\title{
Efficient alignment-free DNA barcode analytics
} Pavel Kuksa and Vladimir Pavlovic*

\author{
Address: Department of Computer Science, Rutgers University, Piscataway, NJ 08854, USA \\ E-mail: Pavel Kuksa - pkuksa@cs.rutgers.edu; Vladimir Pavlovic* - vladimir@cs.rutgers.edu \\ *Corresponding author
}

Published: 10 November 2009

BMC Bioinformatics 2009, I0(Supp| I4):S9 doi: I0.II86/I47I-2I05-I0-SI4-S9

This article is available from: http://www.biomedcentral.com/I47I-2/05/I0/SI4/S9

Publication of this supplement was made possible thanks to sponsorship from the Encyclopedia of Life and the Consortium for the Barcode of Life. (C) 2009 Kuksa and Pavlovic; licensee BioMed Central Ltd.

This is an open access article distributed under the terms of the Creative Commons Attribution License (http://creativecommons.org/licenses/by/2.0), which permits unrestricted use, distribution, and reproduction in any medium, provided the original work is properly cited.

\begin{abstract}
Background: In this work we consider barcode DNA analysis problems and address them using alternative, alignment-free methods and representations which model sequences as collections of short sequence fragments (features). The methods use fixed-length representations (spectrum) for barcode sequences to measure similarities or dissimilarities between sequences coming from the same or different species. The spectrum-based representation not only allows for accurate and computationally efficient species classification, but also opens possibility for accurate clustering analysis of putative species barcodes and identification of critical within-barcode loci distinguishing barcodes of different sample groups.

Results: New alignment-free methods provide highly accurate and fast DNA barcode-based identification and classification of species with substantial improvements in accuracy and speed over state-of-the-art barcode analysis methods. We evaluate our methods on problems of species classification and identification using barcodes, important and relevant analytical tasks in many practical applications (adverse species movement monitoring, sampling surveys for unknown or pathogenic species identification, biodiversity assessment, etc.) On several benchmark barcode datasets, including ACG, Astraptes, Hesperiidae, Fish larvae, and Birds of North America, proposed alignment-free methods considerably improve prediction accuracy compared to prior results. We also observe significant running time improvements over the state-of-the-art methods.

Conclusion: Our results show that newly developed alignment-free methods for DNA barcoding can efficiently and with high accuracy identify specimens by examining only few barcode features, resulting in increased scalability and interpretability of current computational approaches to barcoding.
\end{abstract}

\section{Background}

Identification of living species is one of the pressing tasks in science and technology today, prompted by our need to understand the natural biodiversity and its increasing interaction with the human society.
However, development of comprehensive species identification strategies is impeded by the enormous biodiversity of life on Earth. Traditional morphological identification of species is difficult, requires expertise of highly trained taxonomists, and takes up enormous 
amounts of time. Species identification methods based on molecular diagnostic technologies, including PCR, are limited in the number of species they can identify and lack standardization of technologies or are susceptible to tissue conditions. DNA barcoding has been recently introduced as a taxonomic tool for characterizing species using fragments of a DNA sequence from standard gene regions, such as the mitochondrial DNA (mtDNA) [1]. These relatively short sequences (about 650 symbols in the case of mtDNA) are used as markers for discerning taxonomical identities of specimens using the process of mtDNA extraction, fragment amplification, sequencing and database lookup [2]. A critical property of this particular region is its monophyletic association: the content of mtDNA is often preserved within a species and shows greater divergence between than within species (sometimes $10 \times$ or more when sister species are excluded) [3]. In particular, a region corresponding to $c$ oxidase subunit 1 or cox 1 gene is often used as a critical barcoding marker [1] that exhibits such properties.

Barcoding has shown great promise in practice. DNA barcodes can offer increased adaptability, robustness, and predictive value for rapid and accurate identification of species. For instance, barcoding analysis can result in improved correct placement of previously unknown species or increased resolution of specimens [4], identification of fish products with high accuracy [5], substitutes in fish species for human consumption [6] or marketing of endangered specimens [7]. DNA barcoding has been applied with great initial success to identification across the spectrum of living species, from algea [8], fungi [8], bacteria [9], to plants [10-12], spiders [13], fish [14], birds [1], and rats [15].

Most current barcoding computational methods leverage established modeling approaches from molecular phylogenetic analysis. Traditional barcoding methods, c.f., $[1,16]$, are essentially tree-based phylogenetic approaches where identification decisions are made using a-priory threshold on the tree-induced distances. Choosing an optimal threshold is a challenging task, affected by variable relationship between the species morphology and the cox1 content similarity. More recently, sophisticated Bayesian and decision theory approaches $[17,18]$ have been proposed that attempt to address this problem in a more systematic manner. Traditional phylogenetic methods are also sensitive to the choice of the sequence similarity metrics and the presence of exogenous variations in the sequence (such as those caused by bacterial cosegregation). Moreover, methods of molecular phylogeny are not inherently aimed at the task of sequence delineation, rather the study of relationships at different points in evolutionary history. As a consequence, they can also sometimes exhibit high computational complexity, justified for the complex analysis task but often unnecessary when the goal is e.g., species identification.

More recently, methods that more directly tackle the problem of barcode-based identifications have emerged. Some of these methods, such as [16] use the tools of generic but widely available and highly computationally optimized biological sequence comparison (BLAST or PSI-BLAST). Approach such as [19] even more immediately focuses on the prediction problem. However, a number of challenges remain to be addressed, including the accuracy of identification $[16,18,20,21]$, as well as the efficiency and scalability of computational methods.

In this study we investigate alignment-free kernel methods for the DNA barcoding. Kernel-based classification has demonstrated strong performance in many related tasks of biological sequence analysis, such as protein classification and remote homology detection [22-24]. In the process, a number of kernel types or similarity measures between sequences have been proposed, including kernels derived from probabilistic models [25], $k$-mer string kernels [22,23], and weighted-decomposition kernels [26]. In this work we focus on $k$-mer string kernels, and in particular the spectrum/mismatch kernel methods. In our approach, species identification is performed by first transforming variable-length sequences into fixed-length representations (string spectra) and then classifying resulting spectral representations into one of many established species classes using state-of-the-art classification algorithm (e.g. nearest neighbor or Support Vector Machine (SVM) classifiers $[27,28])$. As a result, the alignment free kernel-based species identification in our study demonstrates both high accuracy, improved speed and classification performance compared to previously employed DNA barcoding identification methods.

\section{Methods}

In this section we discuss alignment-free analytics that we propose to use for accurate and efficient multi-class classification and identification of barcode sequences.

\section{The spectrum kernel methods}

Varying sequence length as well as the warping processes within sequences (insertions/deletions) typically preclude direct application of efficient computational models and algorithms designed for data in Euclidean spaces. The spectrum kernel methods [29,30] resolve this problem using fixed-length representations of arbitrary long sequences. These representations or features describe the statistics of short substrings of length $k$, also known 
as $k$-mers, contained in the original sequence. Such representations are both efficient to compute and informative for the tasks of sequence analysis.

Consider a sequence $X$ of length $n$ represented as a string of symbols $\left(x_{1}, x_{2}, \ldots, x_{n}\right)$ from some alphabet $\Sigma, x_{i} \in \Sigma$. In the case of DNA sequences this alphabet consists of the set of the four DNA bases, $\{A, C, T, G\}$. Spectrum methods construct a fixed-length feature vector $\Phi(X)$ from this arbitrary long sequence by counting the frequencies of occurrence of all $k$-mers $x_{i}, x_{i+1}, \ldots, x_{i+k-1}$ in $X$. This feature, the histogram of $k$-mers in $X$, is commonly referred to as the sequence spectrum. The spectrum's domain has the dimension $|\Sigma|^{k}$ corresponding to the total number of all possible fragments of length $k$ and, as a result, induces a fixed length representation.
This concept is illustrated in Figure 1. A sequence from the Astraptes set is represented as the histogram of frequencies with which 5-long fragments (5-mers) occur in that sequence. In the case of 5-mers there are $\Sigma^{k}=4^{5}=$ 1024 such possible fragments, some of which are identified on the horizontal axes of the count plots in Figure 1. For instance, the fragment "CCGCG" occurs three times. Hence, the Astraptes sequence is mapped to a 1024-dimensional fixed-length representation. This representation will be subsequently used to judge similarities and dissimilarities between pairs of sequences coming from the same or different species.

In practice the spectrum mapping will produce sparse feature vectors of counts when either $k$ is long or the sequences are short. On average and assuming a random sequence generation process, for a sequence of length $n$

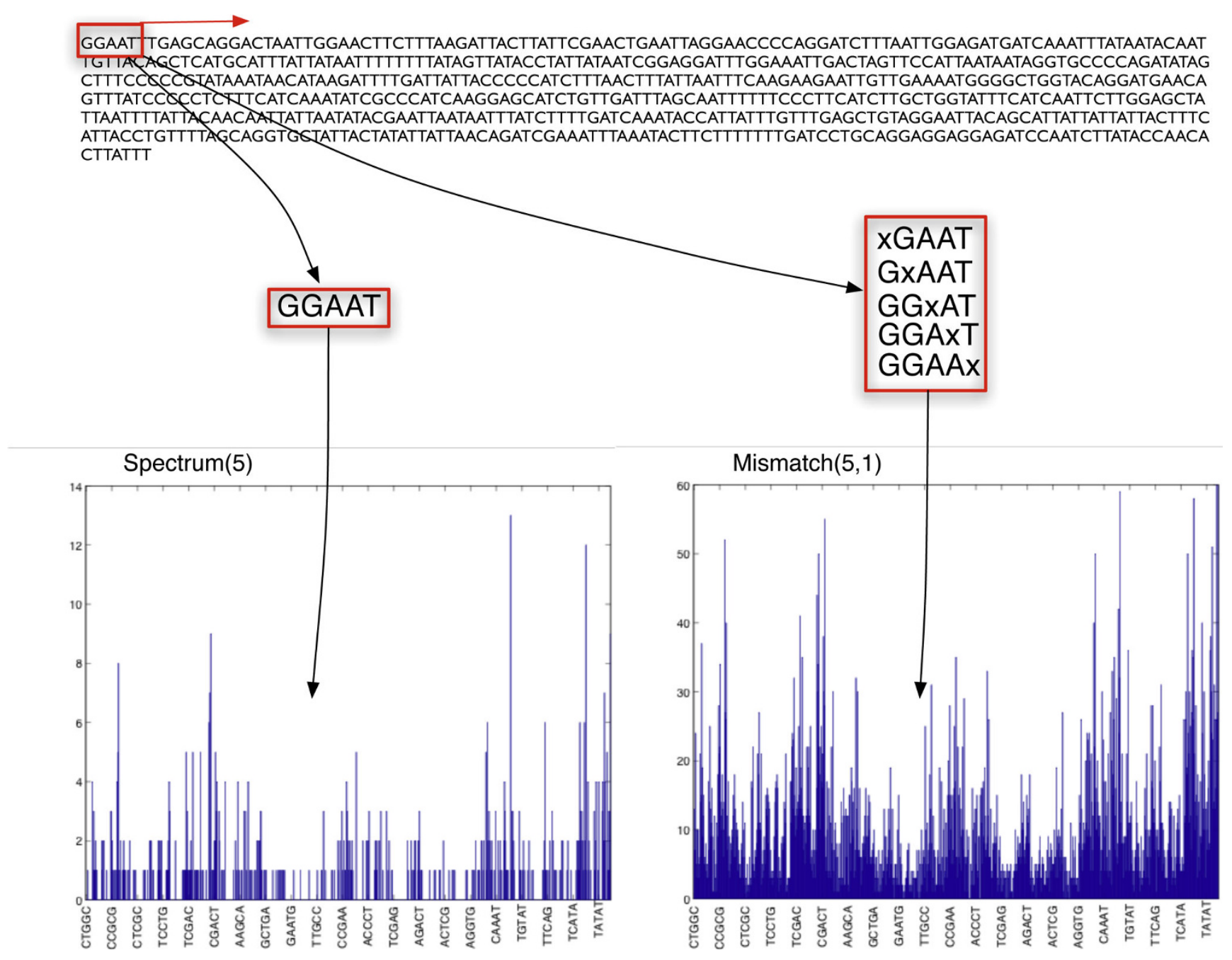

Figure I

Illustration of spectrum and mismatch features. 
each feature will appear $n /|\Sigma|^{k}$ times. While the use of larger $k$ is preferred to yield higher specificity of features, it inadvertently can lead to representations or feature spaces that are too high dimensional and produce low similarity even between sequences in the same class (species). As a consequence, it is often necessary to increase the "density" of these features to allow sufficient within-class sensitivity while maintaining the specificity across classes.

Increasing density for a fixed $k$-mer length can alternatively be viewed as the process of inexact sequence matching. The mismatch kernel method [29] accomplishes this task using the following general mismatch $(k$, m) $|\Sigma|^{k}$-dimensional representation of sequence $X$ :

$$
\Phi^{k, m}(X)=\left(\sum_{\alpha \in X} I_{m}(\alpha, \gamma)\right)_{y \in \Sigma^{k}},
$$

where $I_{m}(\alpha, \gamma)=1$ if $\alpha \in N(\gamma, m)$ and $N(\gamma, m)$ denotes the set of contiguous substrings of length $k$ that differ from $\gamma$ in at most $m$ positions. In other words, in addition to counting all $k$-mers $\alpha$ present in sequence $X$, one also adds counts of $k$-mers that differ in at most $m$ symbols from each $\alpha$. This process is illustrated in Fionure 1 where 5-mer "GGAAT" is mapped to a set of $\left(\begin{array}{l}k \\ m\end{array}\right) \cdot\left(|\Sigma|^{m}-1\right)+1=5 \times 3+1=16$ similar $k$-mers, at most one symbol $(m=1)$ different from "GGAAT". The induced feature vector $\Phi^{k, m}(X)$ has the same dimension as the regular spectrum feature $\Phi(X)$, but is "denser". The choice of the maximum number of the mismatches $(\mathrm{m})$ allowed between any two particular $k$-mers typically depends on whether sequences are relatively similar (e.g. closely related families, $m$ is small) or are far apart (e.g. remote homologs, large values of $m$ may be needed). The exact spectrum kernel is a particular case of the mismatch kernel and can be obtained from Eq. 1 by setting the number of mismatches $m$ to zero (this will result in counting only exact matches between $k$-mers). Both mismatch and exact spectrum methods measure similarity of sequences by comparing the fixed-length features $\Phi^{k, m}$ of those sequences without performing any sequence alignment. As we discuss in the next section, the computational cost of evaluating this similarity is linear in the length of the sequences, compared to quadratic complexity required by alignment-based methods (e.g. Smith-Waterman) for similarity evaluation. This leads to a potentially important advantage for these methods when applied to large DNA barcode sets, which we demonstrate empirically in our Results.

\section{Alignment-free algorithms}

Both mismatch and spectrum methods typically evaluate similarity $K(X, Y)$ of a pair of sequences by computing the dot-product between their corresponding feature vectors (Eq. 1):

$$
K(X, Y)=<\Phi^{k, m}(X), \Phi^{k, m}(Y)>=\sum_{\gamma \in \Sigma^{k}} \sum_{\alpha \in X} I_{m}(\alpha, y) \sum_{\beta \in Y} I_{m}(\beta, \gamma)
$$

Direct evaluation of the dot-product above for similarity computation results in costly $O\left(|\Sigma|^{k} n\right)$ complexity. To efficiently evaluate the dot-product, we first note that in Eq. 2 the product $I_{m}(\alpha, \gamma) I_{m}(\beta, \gamma)$ is non-zero (i.e. contributes to the total similarity/kernel value) only if $\gamma$ is the neighbor for both $\alpha$ and $\beta$. We then write the dotproduct (Eq. 2) as follows:

$$
K(X, Y)=\sum_{\alpha \in X} \sum_{\beta \in Y} \sum_{y \in \Sigma^{K}} I_{m}(\alpha, \gamma) I_{m}(\beta, \gamma)=\sum_{\alpha \in X} \sum_{\beta \in Y} I_{k, m}(\alpha, \beta)
$$

where $I_{k, m}(\alpha, \beta)$ is the number of $k$-mers $\gamma$ shared by $\alpha$ and $\beta$. We observe that the number of shared $k$-mers $I_{k \text {, }}$ ${ }_{m}(\alpha, \beta)$ depends on the Hamming distance (i.e., the number of differences, in symbols, between the strings) $d$ $(\alpha, \beta)$ between $\alpha$ and $\beta$ for a fixed alphabet $\Sigma$, the length of the $k$-mer $k$, and the number of mismatches $m$ (i.e. $I_{k}$, ${ }_{m}(\alpha, \beta)$ can only have a fixed set of values with each value corresponding to a particular Hamming distance). Since the maximum Hamming distance that will result in the non-zero $I_{k, m}(\alpha, \beta)$ is $2 m$, the dot-product in Eq. 3 reduces to computing the number of pairs $(\alpha, \beta), \alpha \in X$, $\beta \in Y$, for each of possible Hamming distances from 0 to $2 m$ :

$K(X, Y)=\sum_{\alpha \in X} \sum_{\beta \in Y} I_{k, m}(\alpha, \beta)=\sum_{d=0}^{2 m} \#$ pairs $(\alpha, \beta), \alpha \in X, \beta \in Y$, at Hamming distanœ $d$

As we show in [31], the mismatch/spectrum similarity measure in the form as in Eq. 4 can be efficiently computed in $O\left(c_{k, m} n\right)$ time, where $c_{k, m}$ is a constant that depends only on the $k$-mer length and the maximum number of allowed differences $m$ but not on the sequence length $n$. In the case of the exact spectrum method, the complexity is $O(k n)$, i.e. is linear in both the sequence length $n$ and the $k$-mer length $k$. It is also important to note that we typically need to evaluate this similarity for a set of $N$ sequences (e.g., DNA barcode samples). Instead of evaluating similarity for every pair of $N$ sequences, a task proportional to $N^{2}$, in [31] we also show that this can be accomplished in the time linear in $N$. Hence, the overall complexity of evaluating the mismatch $(k, m)$ similarity on a set of $N$ sequences of maximal length $n$ is $O\left(c_{k, m} n N\right)$. This results in significant computational savings (speedup) when it is necessary to compute similarity among a large number of sequences, as may be the case with DNA barcodes. 


\section{Prediction models}

Given the similarity kernel for any pair of sequences, one can consider several predictive tasks. One such task is the classification of new sequence samples into one of the previously seen classes. In the context of DNA barcoding, this task can be interpreted as either the classification of a barcode sample into one of the known species or the verification task of resolving whether the sample belongs to a particular species or not. We first consider the latter (verification) task and then generalize it to the full classification task. A very general class of predictive models that relies on the similarity metric induced by the kernel $K$ computes the matching score between the query sequence $X$ and the previously seen sequences $\left\{X_{1}, \ldots\right.$, $\left.X_{N}\right\}$ whose class assignments $\left\{y_{1}, \ldots, y_{N}\right\}$ are known. The score is formed as

$$
f(x)=\sum_{i=1}^{N} w_{i} k\left(x, x_{i}\right)
$$

The sign of this score then typically indicates whether the query $X$ belongs to a particular class, $f(X)>0$, or not. The weights $w_{i}$ are set in a training procedure prior to making predictions using a variety of available "learning" algorithms that attempt to optimize the predictive performance of this model. This verification model can also be generalized to the classification setting, where the sample is to be classified in one of $M$ possible classes. In that case one can construct the predictive model for each class, $f_{m}(X)=\Sigma_{i} w_{m},{ }_{i} K\left(X, X_{i}\right)$, and make the final prediction by finding the class with the maximum score, $y^{*}=\arg \max _{m} f_{m}(X)$.

In this work we consider two classes of algorithms that have generally shown state-of-the-art performance on prediction tasks. One is the simple Nearest Neighbor classifier. In that setting $w_{m, i}$ is non-zero, i.e. $w_{m, i}=1$, only for the sequence $X_{i}$ (of class $y_{i}=m$ ) which is "closest", or most similar, to the query sequence $X$. Nearest neighbor classifiers are simple and have appealing (asymptotic) theoretical properties.

The second class of learning algorithms used in this work is the well-known Support Vector Machine [28]. In the view of the model above the SVM selects an optimal subset of training sequences $X_{i}$ (the so-called support vectors) and sets their weights to maximize the models predictive accuracy. In our work we use the "one-vs-rest" SVM learning approach described in [32].

\section{Results and discussion}

To demonstrate the utility of the alignment-free sequence representation for DNA barcode analytics we primarily focus on the task of species identification. The identification or classification task is one of the relevant analytical problems considered so far in DNA barcoding $[16,18,20,21]$. In this section we show that the spectrumbased, alignment-free representation possesses several interesting properties, among them the high accuracy of the sample-to-species assignments as well as the computational efficiency. Moreover, the spectral representations offer interesting insights into which sequence markers/ features within the standard barcode region (e.g. cox1) serve as the most important discriminants among the sets of species. This result has further implication on computational efficiency but may also facilitate further taxonomical studies. We perform the barcode-based species classification experiments using several benchmark barcode datasets from various barcode collecting campaigns for mammals, fish, birds, lepidoptera, etc. In particular, we use seven data sets of DNA barcodes including Astraptes (12 species), Hesperiidae (364 species), Bats of Guyana (96 species), Fish of Australia (211 species), Birds of North America (656 species), ACG (573 species), and Fish larvae (7 species). Astraptes, Hesperiidae, Bats of Guyana, Birds of North America, and Fish of Australia were compiled from the BOLD [33] project. ACG set was published as a part of [34]. The Fish larvae set appeared in [16]. Table 1 summarizes details of these datasets.

Using these datasets, we consider barcode class prediction problem as a multi-class classification problem described in the Methods section. For the SVM prediction approach, we use one-vs-rest setting to perform the multi-class classification using binary predictors for each class. We evaluate alignment-free similarity of DNA barcodes using the spectrum/mismatch representations of Section 'The spectrum kernel methods' and contrast it to several standard similarity metrics employed for biological sequences and DNA barcodes in particular. In all experiments, we normalize the similarity/ kernel values $K(X, Y)$ using $K^{\prime}(X, Y)=K(X, Y) /$ $\sqrt{K(X, X) K(Y, Y)}$ to remove the dependency between the kernel value and the sequence length. To perform our experiments, we use an existing SVM implementation from a standard machine learning package SPIDER [35] with default parameters. For the spectrum/mismatch

Table I: Barcode datasets

\begin{tabular}{lll}
\hline Dataset & \# species & \# barcodes \\
\hline ACG & 573 & 4267 \\
Hesperiidae & 364 & 2185 \\
Astraptes & 12 & 465 \\
Bats of Guyana & 96 & 840 \\
Birds of North America & 656 & 2589 \\
Fish of Australia & 211 & 754 \\
Fish larvae & 7 & 35 \\
\hline
\end{tabular}


kernel, we use mismatch $(5,1)(k=5$, and $m=1)$ and spectrum-10 $(k=10)$ kernels. To facilitate experiments on large datasets, we use the kernel computation algorithms proposed by Kuksa et al. in [31]. The data and source code used in our experiments are available at the supplementary website [36].

In the following, we first present results on multi-class species identification problem using alignment-free methods. We then focus on the analysis of withinbarcode markers and show the impact of the marker selection on the identification accuracy. To illustrate the ability of the similarity metric to reduce the withinspecies dispersion while maintaining separability of different species we use clustering analysis in the set of experiments following the marker study. Finally, we provide empirical running time analysis of our proposed approach and contrast it with some state-of-the-art methods.

\section{Species identification}

In the species identification experiments, we use the nearest neighbor classifier and the SVM to predict class assignments for query barcodes based on similarity scores computed using alignment-based (Smith-Waterman, Kimura, Hamming) and alignment-free methods (spectrum and mismatch kernels). To discern the predictive ability of different methods we consider a cross-validation setting in which the species data is randomly split into ten disjoint subsets. Nine of the subsets are used to estimate the prediction models which are subsequently evaluated on the remaining set, in a repeated fashion. We report results averaged over the ten folds and show the accuracy variation of each method.

\section{Nearest neighbor approach}

Classification performance for the nearest neighbor approach using alignment-free kernel methods is summarized in Table 2 where we compare cross-validation error rates of the resulting classifiers on the benchmark barcode datasets. We also report results obtained by running PSI-BLAST search with default parameters on these datasets. The results indicate that the alignmentfree spectral method generally shows the highest

Table 2: Nearest neighbor, 10-fold cross-validation error (\%)

\begin{tabular}{llll}
\hline Dataset & PSI-BLAST & spectrum & mismatch \\
\hline ACG & $3.07 \pm 0.68$ & $2.49 \pm 0.87$ & $3.63 \pm 0.65$ \\
Hesperiidae & $4.62 \pm 0.97$ & $3.57 \pm 1.08$ & $4.38 \pm 1.49$ \\
Astraptes & $13.82 \pm 4.42$ & $1.07 \pm 1.81$ & $1.50 \pm 1.99$ \\
Bats & $1.63 \pm 1.22$ & $1.63 \pm 1.22$ & $1.73 \pm 2.01$ \\
Birds & $7.46 \pm 1.90$ & $6.22 \pm 1.50$ & $7.29 \pm 0.96$ \\
Fish Australia & $5.62 \pm 3.31$ & $5.5 \pm 3.27$ & $5.29 \pm 3.34$ \\
Fish larvae & 2.86 & 2.86 & 5.71 \\
\hline
\end{tabular}

classification accuracy. Compared to PSI-BLAST search, the mismatch similarity exhibits similar results, typically inferior to those of the spectral similarity. In Table 3 we show classification performance of the spectrum method for different values of $k$ ( $k$-mer length). The error rates are shown for the nearest neighbor (1-NN) and for 3-NN and $5-\mathrm{NN}$ classifiers. We observe that the nearest neighbor classifier displays the lowest error rates compared to classifiers that use 3 or 5 nearest neighbors for prediction. As we can see from the table, the spectrum method is relatively robust to the choice of the $k$-mer length, with values of $k=8-15$ resulting in the highest classification accuracy. We also note that the experiments show that the smaller $k$ are satisfactory and increasing $k$ much does not increase accuracy significantly; smaller values of the $k$-mer length also have lower computational complexity compared to the larger values of $k$.

In Table 4, we show classification results contrasting the spectral method with nearest neighbor predictors based on more traditional, alignment-based similarity measures. We consider methods based on both global and local alignments. The global alignment on the set of sequences is obtained using the Needleman-Wunsch algorithm with NUC44 scoring matrix and gap opening/ extending penalties set to 8 . These settings result in multiple alignments largely identical to those available via BOLD [33] for the barcode sets of the publicly available projects we use in our evaluation. Given the global alignment, the sequence similarity is scored using two metrics: the Hamming distance ( $0 / 1$ mismatch/ match score) and the Kimura distance. For local alignment-based pairwise scores we use the SmithWaterman scoring with the same parameters as the global alignment model. The results in Table 4 indicate that the alignment-free spectral similarity yields the overall most accurate species predictors. The spectral and Kimura-based distances produce comparable accuracies on three sets (Bats, Birds and Fishes of Australia). As expected, the Hamming $0 / 1$ scoring is typically inferior to other methods as it does not include any measure of varying evolutionary pressures exhibited across different nucleotide pairs. The ACG and the Fish larvae sets are both cases where the spectral method achieves the most accurate prediction among the four contrasted scoring metrics.

To better assess the predictive ability of different measures we also compare the ranking quality of the resulting classifiers on ACG, Hesperiidae, and Birds of North America data sets in Figures 2, 3, and 4, respectively. The ranking score can be used to ascertain how closely the predictions of a model match those of the ideal case as a function of the model specificity. For 
Table 3: Classification performance of the k-spectrum method using nearest neighbor

\begin{tabular}{|c|c|c|c|c|c|}
\hline dataset & $\mathbf{k}=\mathbf{3}$ & $k=5$ & $k=8$ & $k=10$ & $k=15$ \\
\hline \multicolumn{6}{|c|}{ I-NN (nearest neighbor) } \\
\hline ACG & $4.24 \pm 0.90$ & $3.19 \pm 0.93$ & $2.58 \pm 0.94$ & $2.49 \pm 0.87$ & $2.35 \pm 0.83$ \\
\hline Hesperiidae & $5.32 \pm 1.33$ & $4.21 \pm 1.18$ & $3.66 \pm 1.07$ & $3.57 \pm 1.08$ & $3.39 \pm 0.93$ \\
\hline Astraptes & $1.91 \pm 1.87$ & $1.90 \pm 2.08$ & $1.48 \pm 1.75$ & $1.07 \pm 1.81$ & $1.07 \pm 1.81$ \\
\hline Bats of Guyana & $1.87 \pm 1.36$ & $1.63 \pm 1.22$ & $1.63 \pm 1.22$ & $1.63 \pm 1.22$ & $1.63 \pm 1.22$ \\
\hline Birds & $7.77 \pm 1.26$ & $6.68 \pm 1.22$ & $6.42 \pm 1.34$ & $6.22 \pm 1.50$ & $6.13 \pm 1.65$ \\
\hline Fish Australia & $5.47 \pm 3.26$ & $5.35 \pm 3.36$ & $5.35 \pm 3.36$ & $5.50 \pm 3.27$ & $5.50 \pm 3.27$ \\
\hline Fish larvae & 8.57 & 5.71 & 2.86 & 2.86 & 2.86 \\
\hline \multicolumn{6}{|l|}{$3-N N$} \\
\hline ACG & $10.20 \pm 1.31$ & $8.98 \pm 1.23$ & $8.54 \pm 1.11$ & $8.67 \pm 1.33$ & $8.63 \pm 1.21$ \\
\hline Hesperiidae & $15.55 \pm 1.25$ & $14.30 \pm 1.46$ & $14.22 \pm 1.50$ & $14.40 \pm 1.85$ & $14.30 \pm 2.00$ \\
\hline Astraptes & $2.78 \pm 2.49$ & $2.36 \pm 2.16$ & $2.36 \pm 2.16$ & $2.15 \pm 2.29$ & $1.70 \pm 1.96$ \\
\hline Bats of Guyana & $3.77 \pm 1.68$ & $4.46 \pm 2.06$ & $4.35 \pm 2.01$ & $4.46 \pm 2.06$ & $4.46 \pm 2.06$ \\
\hline Birds & $20.23 \pm 2.64$ & $19.58 \pm 2.48$ & $18.88 \pm 2.29$ & $18.99 \pm 2.22$ & $18.37 \pm 2.07$ \\
\hline Fish Australia & $12.44 \pm 5.67$ & $12.32 \pm 5.68$ & $|2.3| \pm 5.38$ & $12.42 \pm 5.46$ & $11.93 \pm 5.06$ \\
\hline Fish larvae & 14.29 & 14.29 & 11.43 & 11.43 & 11.43 \\
\hline \multicolumn{6}{|l|}{$5-N N$} \\
\hline$A C G$ & $|3.4| \pm 2.00$ & $12.42 \pm 1.40$ & $11.49 \pm 1.28$ & $11.49 \pm 1.25$ & $11.28 \pm 1.20$ \\
\hline Hesperiidae & $19.70 \pm 1.71$ & $19.64 \pm 2.62$ & $18.63 \pm 2.21$ & $18.54 \pm 2.17$ & $18.22 \pm 2.17$ \\
\hline Astraptes & $3.43 \pm 3.09$ & $3.01 \pm 2.76$ & $2.14 \pm 1.74$ & $1.70 \pm 1.96$ & $1.06 \pm 1.12$ \\
\hline Bats of Guyana & $6.09 \pm 3.01$ & $5.85 \pm 3.23$ & $5.73 \pm 2.77$ & $5.87 \pm 2.94$ & $5.61 \pm 2.79$ \\
\hline Birds & $27.32 \pm 2.50$ & $26.26 \pm 2.17$ & $26.49 \pm 2.11$ & $26.42 \pm 2.44$ & $26.10 \pm 2.36$ \\
\hline Fish Australia & $19.40 \pm 5.91$ & $18.85 \pm 6.15$ & $19.28 \pm 5.28$ & $18.36 \pm 5.04$ & $|8.8| \pm 4.64$ \\
\hline Fish larvae & 22.86 & 22.86 & 22.86 & 22.86 & 22.86 \\
\hline
\end{tabular}

Table 4: Nearest neighbor performance (10-fold cross-validation error, \%)

\begin{tabular}{lllll}
\hline Dataset & Spectrum & Hamming & Kimura & Smith-Waterman \\
\hline ACG & $\mathbf{2 . 4 9} \pm \mathbf{0 . 8 7}$ & $11.44 \pm 1.52$ & $5.51 \pm 0.86$ & $3.66 \pm 0.66$ \\
Hesperiidae & $\mathbf{3 . 5 7} \pm \mathbf{I . 0 8}$ & $14.49 \pm 2.36$ & $3.81 \pm 1.26$ & $5.45 \pm 1.20$ \\
Astraptes & $\mathbf{1 . 0 7} \pm \mathbf{I . 8 1}$ & $3.61 \pm 2.77$ & $1.71 \pm 1.96$ & $1.64 \pm 1.03$ \\
Bats Guyana & $\mathbf{1 . 6 3} \pm \mathbf{I . 2 2}$ & $2.72 \pm 1.83$ & $\mathbf{1 . 6 3} \pm \mathbf{1 . 2 2}$ & $1.63 \pm 1.22$ \\
Birds of North America & $\mathbf{6 . 2 2} \pm \mathbf{I . 5 0}$ & $18.38 \pm 2.05$ & $\mathbf{6 . 0 2} \pm \mathbf{1 . 3 6}$ & $8.20 \pm 1.53$ \\
Fish Australia & $\mathbf{5 . 5 0} \pm \mathbf{3 . 2 7}$ & $5.87 \pm 4.01$ & $\mathbf{5 . 3 5} \pm \mathbf{3 . 3 6}$ & $5.35 \pm 3.36$ \\
Fish larvae & $\mathbf{2 . 8 6}$ & 11.43 & 8.57 & 5.71 \\
\hline
\end{tabular}

${ }^{\dagger}$ leave-one-out validation error is reported

instance, the top-3 error rate reports the accuracy of prediction if one assumes that correct prediction is made whenever the true species class of a sequence is anywhere among the top-3 scoring classes predicted by a model. Top-1 error rate corresponds to the standard error rate. The higher the $q$, the lower the top- $q$ errors are, at the expense of the specificity of predictions. For good models/similarity measure the ranking error rate typically drops off quickly. In our evaluations we observe that the alignment-free spectrum method consistently shows lower top- $q$ error rates $(n=1 \ldots 10)$ compared to that of alignment-based (Kimura, Smith-Waterman, Hamming) scoring methods (Figures 2, 3, and 4), with the Kimura score approaching the spectrum for intermediate values of $n$ on the sets where the two initially differ. On the ACG set, for instance, the Kimura distance-based scoring becomes comparable to the spectrum measure for $n=4$, which suggests that further tuning of the score parameters may improve Kimura performance. However, doing so would require more complex, most likely heterogeneous, sequence models.

The above sets of experiments indicate that the alignment-free spectral measure may be well-suited for the DNA-barcode based species prediction tasks. In contrast with the alignment methods, the spectral alignment-free scores leave out the need for sometimes complex and sensitive global alignments. The benefit becomes more 


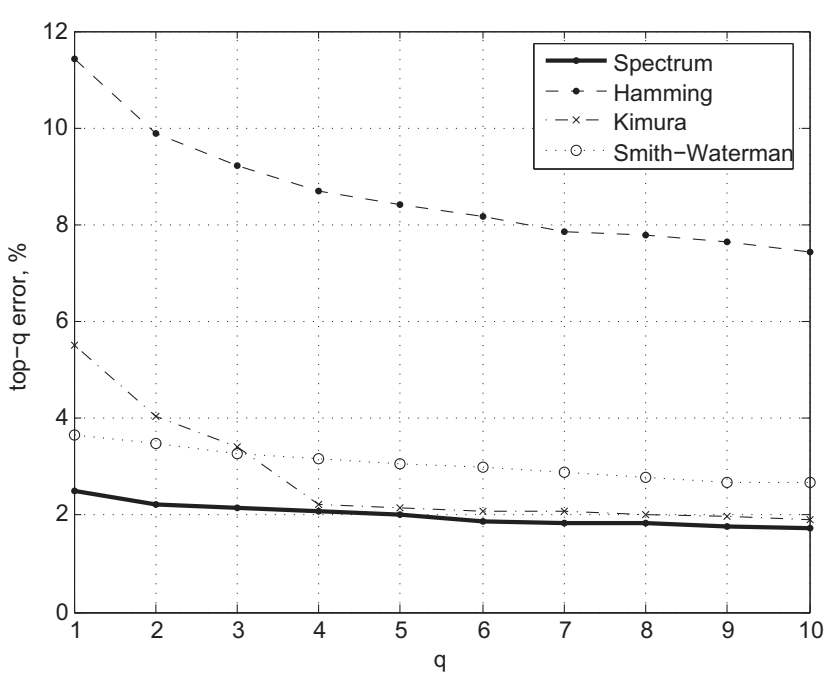

Figure 2

Ranking quality. Comparison of top-q error rates on ACG dataset for the spectrum (alignment-free) method and alignment-based methods.

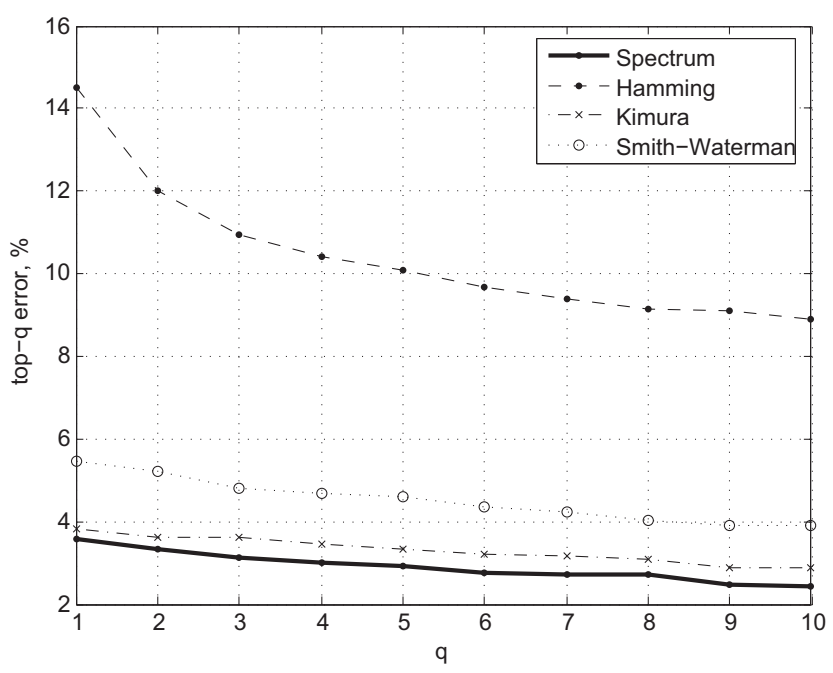

Figure 3

Ranking quality. Comparison of top-q error rates on Hesperiidae dataset for the spectrum (alignment-free) method and alignment-based methods.

significant if one keeps in mind that any addition of new sequences to the set may require (global) re-alignments within a set, the step not necessary in the case of spectrum scores.

One other particularly interesting conclusion is that the spectral methods are better than or similar to the alignment-based scoring metrics even when those metrics take into account differences in evolutionary

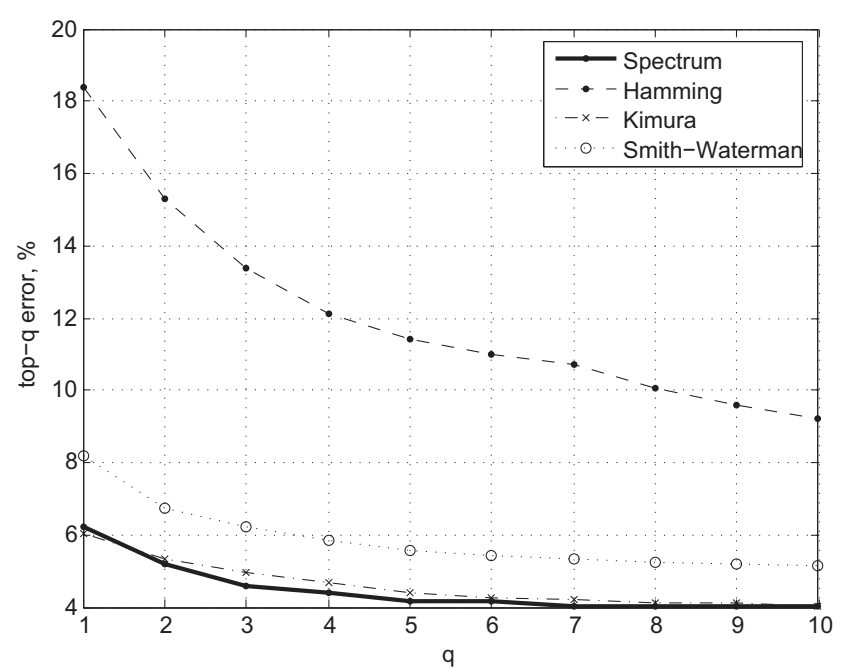

Figure 4

Ranking quality. Comparison of top- $q$ error rates on Birds dataset for the spectrum (alignment-free) method and alignment-based methods.

pressures across different sequence symbols, either imposed by the alignment parameters or the scoring such as that of the Kimura model. This observation should be placed in the context of our alignment-free methods that use the $0 / 1$ scoring but within the short sequence fragments only. Using such scoring on the full sequence scale, as in the Hamming distance measure above, is bound to produce inaccurate species matches. Another important conclusion is that in the case of barcodes the mismatch alignment-free measures generally result in similarities that less accurately model the distribution of sequences within and across different species compared to the exact spectrum methods. This is in contrast with other data domains where the mismatch features have been successfully applied, such as that of the protein sequences [31]. Two factors play a role in this discrepancy. The protein sequences, from the point of our mathematical representation, live in higher-dimensional spaces due to the increased alphabet size (20 vs. 4 ) and hence may require a looser notion of matching. Another factor is the variability within classes (species in barcodes and, e.g., superfamilies in proteins); protein sequences with the same class typically exhibit much higher primary sequence variability than do the DNA barcodes within taxonomic groups. As a consequence, the mismatch measures may not be deemed necessary for the DNA barcoding analytics. A final comment relates to the complexity of computing the proposed measures in conjunction with their predictive performance. As we will demonstrate in Section 'Experimental running time analysis', the alignment-free spectral methods generally incur significant computational advantage over the 
competing alignment-based measures. This can be a deciding factor when the methods are applied to large barcode sets or in instances when new samples become available and the (global) re-alignment is not desirable.

\section{SVM-based classification}

SVM-based classifiers are typically regarded as state-ofthe-art predictors across a wide span of modeling problems. We therefore explore the use of this class of models in the DNA barcode setting. Table 5 displays cross-validation error rate of the SVM classifier on the barcode sets in our study for the two alignment-free scoring metrics of interest. The results demonstrate that using SVM classifiers with alignment-free methods results in similar performance compared to the previously examined nearest neighbor approach. We observe slight but insignificant improvements in average error rates for the spectrum method on ACG, Hesperiidae, and Astraptes data sets, e.g. error rate reduces from 1.07 to 0.86 on Astraptes data set. These results are not unexpected, given the relatively large number of classes (species) in these settings as well as the already low error rates of the nearest neighbor predictor. At the same time, the results suggest that the spectral scoring metric for DNA barcodes appears to fairly accurately reflect the sequence diversity within and between species, and is not significantly affected (nor can be further shaped) by the choice of the predictor/classifier algorithm. We finally note that in our experiments which are not reported here we observed that other measures, which rely on global or local alignments such as the ones in Section 'Nearest neighbor approach', are similarly not affected by the choice of classification models.

Table 5: SVM 10-fold cross-validation error rate (\%)

\begin{tabular}{lll}
\hline Dataset & spectrum $(\mathbf{k}=\mathbf{~ 1 0 )}$ & mismatch \\
\hline ACG & 2.32 & 3.48 \\
Hesperiidae & 3.25 & 3.36 \\
Astraptes & 0.86 & 1.07 \\
Bats & 1.63 & 1.67 \\
Birds & 5.99 & 7.09 \\
Fish Australia & 5.35 & 5.35 \\
Fish larvae & 2.86 & 5.71 \\
\hline
\end{tabular}

\section{Comparison with previously published results}

We observe that alignment-free methods considerably improve identification accuracy compared to the previously reported results of $[18,20]$. For example, on Astraptes dataset [37], the test error rate of the multi-class SVM is only $0.86 \%$ compared to $9 \%$ in [20] or $20 \%$ in [18]. These results further signify the potential of the proposed measures as applied to the barcoding-based prediction task as well as to barcode analytics in general.

\section{Barcode marker selection}

DNA barcodes provide full-length barcode sequences for barcode analytics. However, there are situations where only certain (few) markers within the barcode may be sufficient to accurately and rapidly perform the analytic task. Species prediction based on barcodes, and in particular for the case of a limited number of species, is one such task. The use of markers instead of the full sequence may increase the robustness of predictions by eliminating the potentially irrelevant portions of the barcodes that can also contain errors in barcode data collection. Identification of sequence markers can be also interesting from the perspective of further withinsequence loci analysis. Finally, the use of few markers can also be advantageous from the computational perspective. In this section, we evaluate the marker or feature selection performance using alignment-free methods.

In our experiments, we use the RELIEF [38] feature selection algorithm to find subsets of discriminative spectral markers. Using the $k$-mer or fragment notation we introduced previously, the markers correspond to those $k$-mers most relevant for species identification. Table 6 displays error rates of the nearest neighbor classifiers as a function of the number of selected markers.

As evident from the table, using marker selection results in the performance similar to that when full barcode sequences are used. Even when only few (about 200500) markers are selected the alignment-free methods still result in accurate prediction and perform on-par

Table 6: Feature selection performance using alignment-free methods (error, \%)

\begin{tabular}{lccccccc}
\hline Dataset & & \multicolumn{2}{c}{ \# features selected } & & \\
& Full feature set (1048576 feat.) & $\mathbf{4 0 9 6}$ & $\mathbf{2 0 4 8}$ & $\mathbf{1 0 2 4}$ & $\mathbf{5 I 2}$ & $\mathbf{2 0 0}$ & $\mathbf{1 0 0}$ \\
\hline ACG & $2.49 \pm 0.87$ & $2.51 \pm 0.95$ & $2.79 \pm 1.02$ & $3.00 \pm 0.96$ & $3.17 \pm 0.86$ & $3.52 \pm 0.64$ & $4.48 \pm 0.86$ \\
Hesperiidae & $3.57 \pm 1.08$ & $3.53 \pm 1.12$ & $3.80 \pm 1.22$ & $4.17 \pm 1.05$ & $4.40 \pm 1.15$ & $4.81 \pm 1.30$ & $5.64 \pm 1.20$ \\
Astraptes & $1.07 \pm 1.81$ & $0.44 \pm 0.92$ & $0.44 \pm 0.92$ & $0.44 \pm 0.92$ & $0.44 \pm 0.92$ & $0.64 \pm 1.03$ & $1.49 \pm 1.75$ \\
Bats of Guyana & $1.63 \pm 1.22$ & $1.63 \pm 1.22$ & $1.63 \pm 1.22$ & $1.63 \pm 1.22$ & $1.63 \pm 1.22$ & $1.63 \pm 1.22$ & $1.63 \pm 1.22$ \\
Birds & $6.30 \pm 1.80$ & $6.45 \pm 1.82$ & $6.94 \pm 2.08$ & $7.13 \pm 2.05$ & $7.41 \pm 1.77$ & $9.10 \pm 1.64$ & $9.84 \pm 1.99$ \\
Fish of Australia & $5.50 \pm 3.27$ & $5.35 \pm 3.36$ & $5.35 \pm 3.36$ & $6.14 \pm 3.50$ & $6.80 \pm 3.15$ & $8.32 \pm 2.75$ & $9.51 \pm 2.40$ \\
Fish larvae & 2.86 & 0 & 0 & 0 & 0 & 0 & 0 \\
\hline
\end{tabular}


with alignment-based methods, while being computationally more efficient, as we show in Section Experimental running time analysis. Note that the number of selected markers (e.g., 100) is the total number of marker per set of all sequences and species. The average number of markers per sequence is typically between $20 \%$ and $40 \%$ of the total number of markers (i.e., when top 500 markers are selected, each sequence is represented with about $100-200$ loci, a reduction of $70 \%$ to $85 \%$ from the approximately 650 bases of the full barcode). It is also interesting to note that selecting a smaller set of markers instead of the full barcodes can sometimes lead to more robust and accurate identification. For instance, in the case of Fish larvae set the use of fewer features increases the accuracy. One reason for this may be the presence of distractors or sequencing errors that are eliminated when only the most critical markers are selected.

Figures 5, 6, 7, 8, 9, 10 show, for various barcode sets, the sequence-marker location maps for the top-100 spectrum features (highlighted in different colors). These marker location maps display how the identified top features are positioned within the barcodes. In the figures, the vertical axis corresponds to individual barcode sequences and the horizontal axis corresponds to the position within the aligned (for visualization purpose only) sequences. The blue horizontal lines in the figures indicate species class boundaries, while the color bars next to the maps indicate the color-to-marker index correspondences. For completeness Tables 7, 8, 9, $10,11,12$ show the corresponding top-100 spectrum markers, ranked by the weights assigned by the feature selection algorithm.

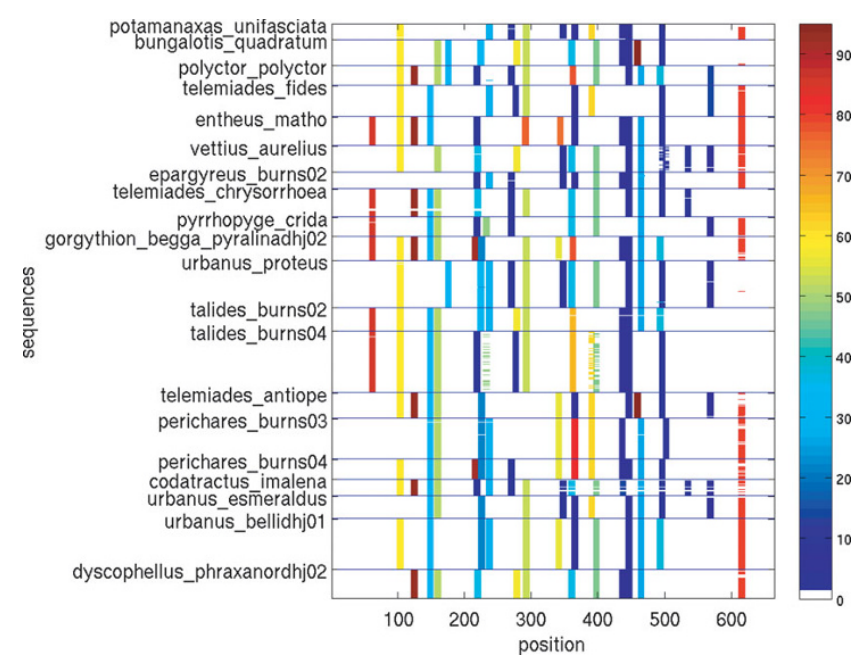

Figure 5

Species sequence map for Hesperiidae data set (top I 00 spectrum $(k=10)$ features).

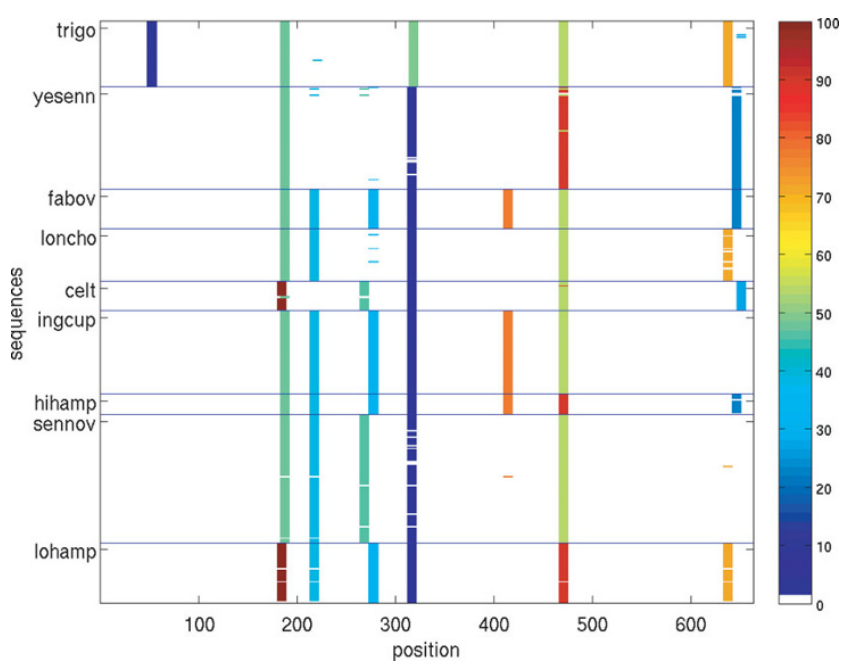

Figure 6

Species sequence map for Astraptes data set (top 100 spectrum $(k=10)$ features).

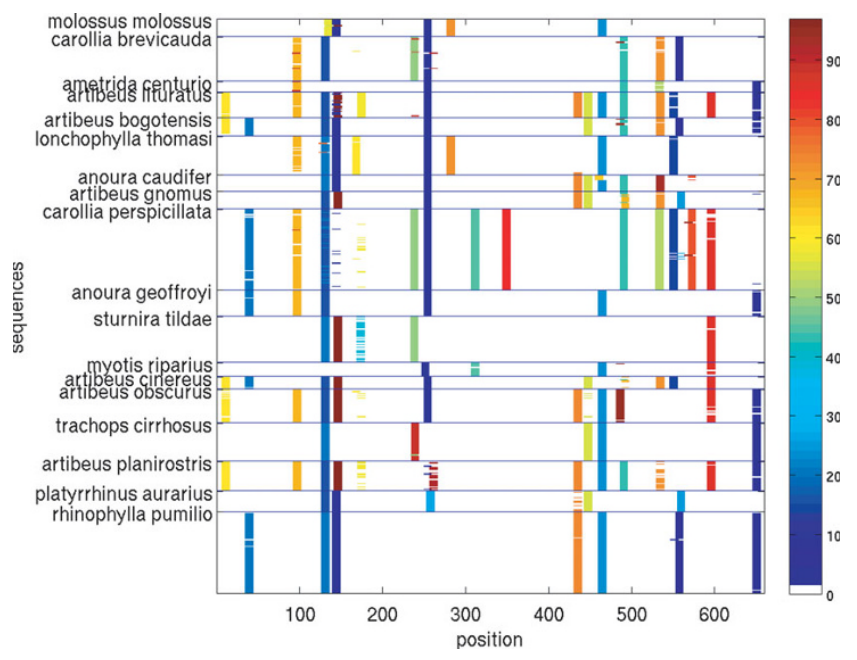

Figure 7

Species sequence map for Bats of Guyana data set (top 100 spectrum $(k=10)$ features).

The results indicate that different species classes have distinct distributions (in terms of both spectrum marker types and their locations) of the first few spectrum markers (top 100 shown). This allows one to accurately distinguish species between each other by solely using the marker sets. As a consequence, these small sets of features can serve as signatures for efficient and accurate classification and identification, among the set of considered species. 


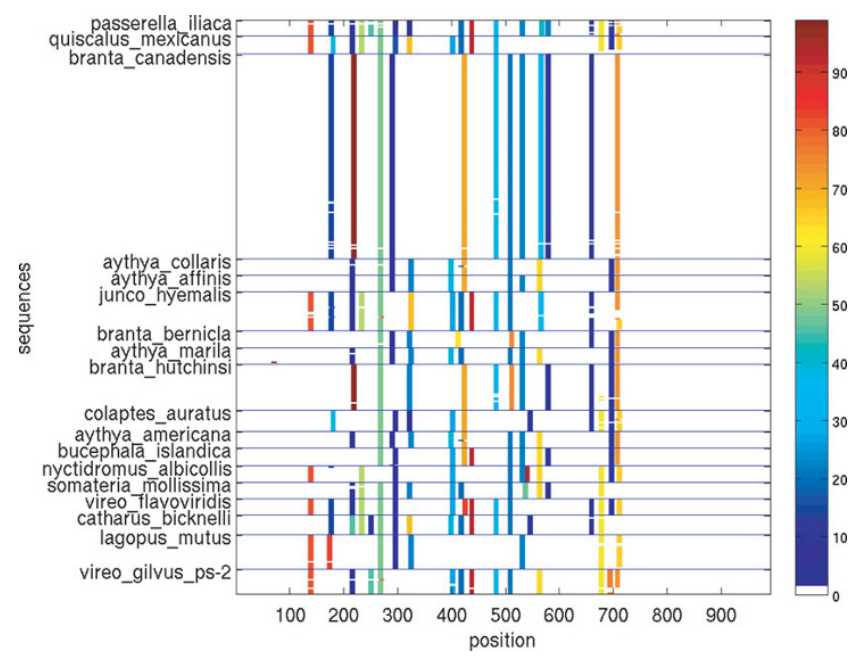

Figure 8

Species sequence map for Birds of North America data set (top 100 spectrum $(k=10)$ features).

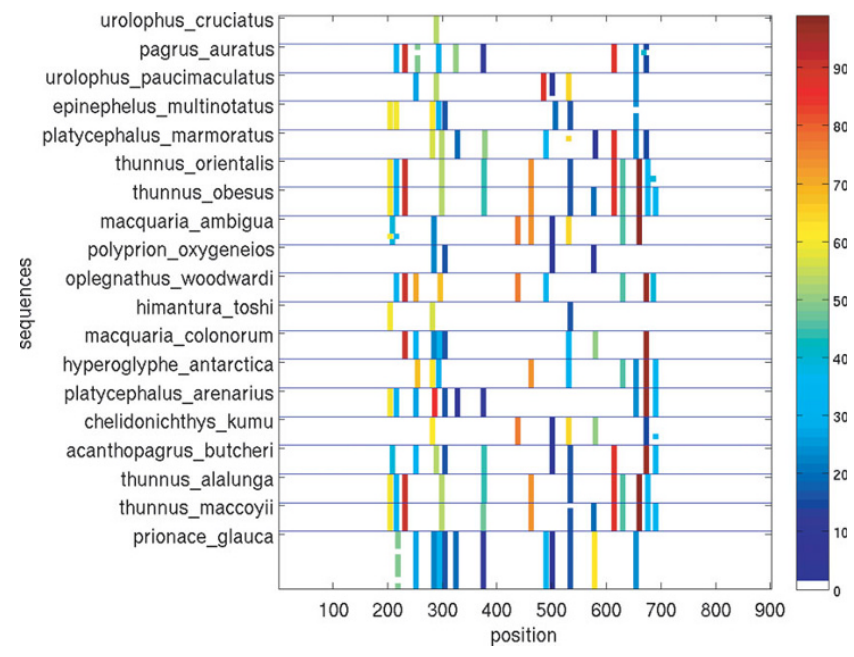

Figure 9

Species sequence map for Fish of Australia data set (top 100 spectrum $(k=10)$ features).

\section{New species detection}

In the classification task that we considered in previous sections, a species label for a new barcode is predicted according to the class (species) models learned from training examples. In this section we consider the problem of identifying whether a barcode belongs to a species in the training set or to a new, unknown species.

We simulate the new species detection by holding out barcode samples from one of the species in the barcode

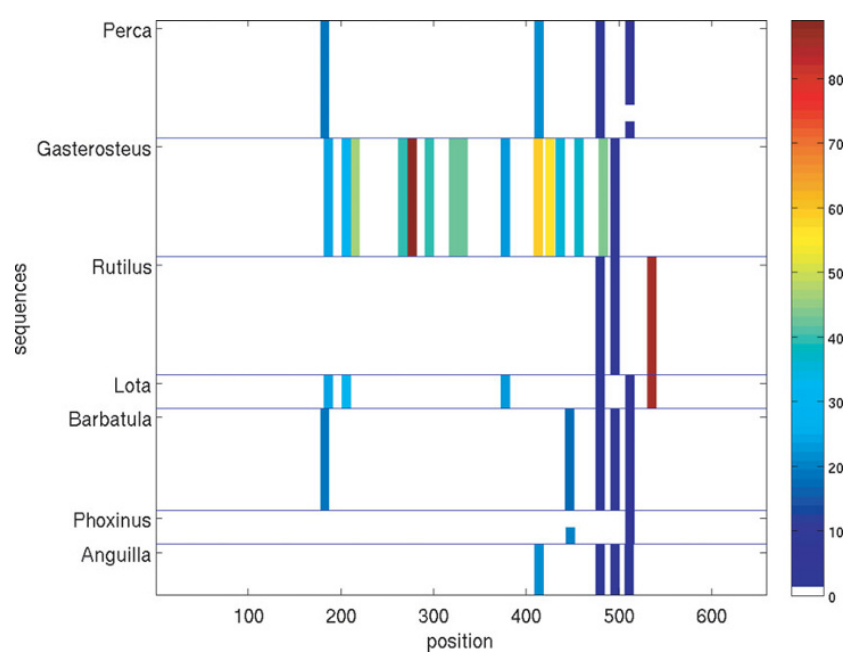

Figure 10

Species sequence map for Fish larvae data set (top 100 spectrum $(k=10)$ features).

dataset. Barcode samples belonging to this species are then presented to the model that only contains barcodes from the remaining "known" species. The query barcode is labeled as a new species if its distance to the nearest barcode in the "known species" set exceeds the average distance between barcodes in the class of that closest known species barcode. Otherwise, the query barcode is assigned to the class of the closest known species. We then measure the new species detection error rate by holding out each species in the dataset and averaging the individual species error rates. The average new species detection error rates are reported in Table 13. The error rates in Table 13 are, thus, the errors from assigning a sample from held-out classes (i.e. new species) to the existing train classes (known species). Table 14 shows new species detection error rates in a slightly more comprehensive but also more realistic task, where we held-out not only the barcodes from the "unknown" species but also some random barcodes from the known species. Hence, the error rates in Table 14 include errors from incorrectly assigning the "new species" label to the barcode samples from one of the known classes but also the errors from assigning the query barcode from new species to one of the existing classes.

As we can see from the results, using the nearest neighbor approach results in about $85-90 \%$ correct identification of the samples belonging to new species. To further investigate the errors committed here we show a slightly more informative representation of the new species detection results in Figure 11. The black curve in each panel indicates the number of classes (vertical axis) with the detection accuracy equal to or higher than the 
Table 7: Top- 100 spectrum features for Hesperiidae data set

\begin{tabular}{|c|c|c|c|c|c|c|c|}
\hline rank & feature & rank & feature & rank & feature & rank & feature \\
\hline I & TTATTATTAT & 26 & ATTAATATAC & 51 & TTTTTATAGT & 76 & GGAGCCCCTG \\
\hline 2 & ATTATTATTA & 27 & CTTTCCCCCG & 52 & TTTTTTATAG & 77 & TATTAATTTC \\
\hline 3 & TATTACСССС & 28 & GCTTTCCCCC & 53 & ATTAATTTCA & 78 & AATATTGCTC \\
\hline 4 & СССССТСТТТ & 29 & TTAATATACG & 54 & TAATATACGA & 79 & TTTTTGATCC \\
\hline 5 & AATTTTATTA & 30 & TATTATAATT & 55 & GTTTATCCCC & 80 & TTTTTTGATC \\
\hline 6 & AGGAGCTATT & 31 & AGCTTTCCCC & 56 & TTTTTTTATA & 81 & ATATTGCTCA \\
\hline 7 & ATTGCCCATC & 32 & GCTCCTGATA & 57 & ССТТСТTТАА & 82 & GAGCCCCTGA \\
\hline 8 & TTGCCCATCA & 33 & TAATTTTATT & 58 & TGGAGATGAT & 83 & ATTGCTCATC \\
\hline 9 & TTAGGAGCTC & 34 & СTCCTGATAT & 59 & TTATTACCCC & 84 & TGCTCATCAA \\
\hline 10 & TCAAATACСТ & 35 & TCCTGATATA & 60 & TTTATССССС & 85 & TTGCTCATCA \\
\hline II & АССТTТАТTТ & 36 & CTAATATTGC & 61 & ССССТСТТТС & 86 & ATTATTAATT \\
\hline 12 & TAGGAGCTCC & 37 & TAGGAGCCCC & 62 & ATTTAGCAAT & 87 & CTCATCAAGG \\
\hline 13 & ATTTTATTAC & 38 & TGATCAAATA & 63 & GATTTAGCAA & 88 & GCTCATCAAG \\
\hline 14 & GGAGCTATTA & 39 & TTAATTTTAT & 64 & TATTATTATT & 89 & TACСССССТС \\
\hline 15 & ATTAGGAGCT & 40 & TTTGATCAAA & 65 & AGGAGCTCCT & 90 & TAGCTTTCCC \\
\hline 16 & TATTGCCCAT & 41 & TTGATCAAAT & 66 & AATATTGCCC & 91 & ATATTAGGAG \\
\hline 17 & CAAATACCTT & 42 & CTTTATTTGT & 67 & ATATTGCCCA & 92 & TATTAGGAGC \\
\hline 18 & TGCCCATCAA & 43 & CCTTTATTTG & 68 & ATTATTAATA & 93 & TACTATTGTT \\
\hline 19 & AАТАССТTТА & 44 & TATTAATATA & 69 & ATTATTACCC & 94 & ATAGCTTTCC \\
\hline 20 & AAATACCTTT & 45 & ATTAATTTTA & 70 & TTATTAATAT & 95 & CAATTATTAA \\
\hline 21 & СCСCTGATAT & 46 & TACCTTTATT & 71 & AGATGATCAA & 96 & ACAATTATTA \\
\hline 22 & GCCCCTGATA & 47 & AATTTTTTCT & 72 & GAGATGATCA & 97 & TTTAGCAATT \\
\hline 23 & CCCATCAAGG & 48 & TATAGCTTTC & 73 & GGAGATGATC & 98 & CCCCCGAATA \\
\hline 24 & CCCTGATATA & 49 & AGCCCCTGAT & 74 & TTTTATAGTT & 99 & TCCCCCGAAT \\
\hline 25 & GCCCATCAAG & 50 & ATACCTTTAT & 75 & TTACСССССТ & 100 & TTCCCCCGAA \\
\hline
\end{tabular}

Table 8: Top- 100 spectrum features for Astraptes data set

\begin{tabular}{|c|c|c|c|c|c|c|c|}
\hline rank & feature & rank & feature & rank & feature & rank & feature \\
\hline 1 & TATACCAACA & 26 & СТTATATCAA & 51 & AATGGAGCTG & 76 & GGAGGAGACC \\
\hline 2 & TTATACCAAC & 27 & TATATCAACA & 52 & ATGGAGCTGG & 77 & ATCTTGCCGG \\
\hline 3 & TGAAAATGGA & 28 & TATCAACACT & 53 & GAAAATGGAG & 78 & CATCTTGCCG \\
\hline 4 & AACTTCTTTA & 29 & TCAACACTTA & 54 & AATATACGAA & 79 & TCATCTTGCC \\
\hline 5 & ACTTCTTTAA & 30 & TCTTATATCA & 55 & ATATACGAAT & 80 & CCGGTATTTC \\
\hline 6 & СТTCTTTAAG & 31 & TTATATCAAC & 56 & ATTAATATAC & 81 & CGGTATTTCA \\
\hline 7 & СTTTAAGATT & 32 & АСССССАТСТ & 57 & TAATATACGA & 82 & CTTGCCGGTA \\
\hline 8 & TCTTTAAGAT & 33 & ATTACССССA & 58 & TATACGAATT & 83 & GCCGGTATTT \\
\hline 9 & TTCTTTAAGA & 34 & ATTATTACCC & 59 & TATTAATATA & 84 & TCTTGCCGGT \\
\hline 10 & TTTAAGATTA & 35 & TACССССATC & 60 & TTAATATACG & 85 & TGCCGGTATT \\
\hline II & GAACTTCTTT & 36 & TATTACСССC & 61 & AAAATGGGGC & 86 & TTGCCGGTAT \\
\hline 12 & GGAACTTCTT & 37 & TTACССССAT & 62 & AAATGGGGCT & 87 & ATACGAATTA \\
\hline 13 & TGGAACTTCT & 38 & TTATTACCCC & 63 & AATGGGGCTG & 88 & TACGAATTAA \\
\hline 14 & AATCTTATAC & 39 & AATAATAGGT & 64 & ATGGGGCTGG & 89 & ACGAATTAAT \\
\hline 15 & ACCAACACTT & 40 & AATAGGTGCC & 65 & GAAAATGGGG & 90 & AATATGCGAA \\
\hline 16 & ATACCAACAC & 41 & AGGTGCCCCA & 66 & GGCTGGTACA & 91 & ATATGCGAAT \\
\hline 17 & ATCTTATACC & 42 & ATAGGTGCCC & 67 & GGGCTGGTAC & 92 & ATGCGAATTA \\
\hline 18 & CCAACACTTA & 43 & GGTGCCCCAG & 68 & GGGGCTGGTA & 93 & ATTAATATGC \\
\hline 19 & СТTATACCAA & 44 & GTGCCCCAGA & 69 & TGAAAATGGG & 94 & GCGAATTAAT \\
\hline 20 & TACCAACACT & 45 & TAGGTGCCCC & 70 & TGGGGCTGGT & 95 & TAATATGCGA \\
\hline 21 & TCTTATACCA & 46 & TTGATTATTA & 71 & AGGAGACCCA & 96 & TATGCGAATT \\
\hline 22 & AATCTTATAT & 47 & TGGAGGATTT & 72 & AGGAGGAGAC & 97 & TATTAATATG \\
\hline 23 & ATATCAACAC & 48 & TGCCCCAGAT & 73 & GAGACCCAAT & 98 & TGCGAATTAA \\
\hline 24 & ATCAACACTT & 49 & AAAATGGAGC & 74 & GAGGAGACCC & 99 & TTAATATGCG \\
\hline 25 & АTCTTATATC & 50 & AAATGGAGCT & 75 & GGAGACCCAA & 100 & AATTGGAGGA \\
\hline
\end{tabular}


Table 9: Top-100 spectrum features for Bats of Guyana data set

\begin{tabular}{|c|c|c|c|c|c|c|c|}
\hline rank & feature & rank & feature & rank & feature & rank & feature \\
\hline I & ATAATTGGAG & 26 & AGCTTCTGAC & 51 & TTTCCCCGAA & 76 & GTAACAGCCC \\
\hline 2 & TTGTAATAAT & 27 & CCTGTCCTAG & 52 & GTCTTATTAC & 77 & TAACAGCCCA \\
\hline 3 & TTTGTAATAA & 28 & CTGTCCTAGC & 53 & TCTTATTACT & 78 & TGTTCTAGCA \\
\hline 4 & ATAAGCTTCT & 29 & GCTTCTGACT & 54 & AGCCCATGCC & 79 & ATCACTATAC \\
\hline 5 & TAAGCTTCTG & 30 & GTTCTAGCAG & 55 & TTATTACTAC & 80 & TCACTATACT \\
\hline 6 & GTCCTAGCAG & 31 & TGTCCTAGCA & 56 & CCCATGCCTT & 81 & AAGCAGGAGT \\
\hline 7 & TCCTAGCAGC & 32 & TTCTAGCAGC & 57 & GCCCATGCCT & 82 & САСТАТАСТА \\
\hline 8 & CAACACTTAT & 33 & TCCTGTCCTA & 58 & TTATAATTGG & 83 & ACACTTATTC \\
\hline 9 & CCTAGCAGCA & 34 & ATAGTAGGCA & 59 & ATTATAATTG & 84 & CCTAGCAGGC \\
\hline 10 & AATATAAGCT & 35 & GTAACAGCTC & 60 & TATAATTGGA & 85 & AAACCTTAAT \\
\hline II & ATATAAGCTT & 36 & TAACAGCTCA & 61 & AACAGCCCAT & 86 & AАССТTAATA \\
\hline 12 & CTTCCTGTCC & 37 & AACAGCTCAT & 62 & TACCTATTAT & 87 & ACСТTAATAC \\
\hline 13 & TTCCTGTCCT & 38 & ATCATAATTG & 63 & CCTGTTCTAG & 88 & TATTAGGTGA \\
\hline 14 & AAGCTTCTGA & 39 & ATCAACACTT & 64 & CTGTTCTAGC & 89 & CCCCGAATAA \\
\hline 15 & TCTTCCTGTT & 40 & TATCAACACT & 65 & TATTAATATA & 90 & CCCGAATAAA \\
\hline 16 & ACAGCTCATG & 41 & TCAACACTTA & 66 & TTTATTACTA & 91 & TCTGACTCCT \\
\hline 17 & CAGCTCATGC & 42 & TCATAATTGG & 67 & ATCAAACACC & 92 & TTCTGACTCC \\
\hline 18 & AACACTTATT & 43 & ATATCAAACA & 68 & ATTAGGTGAT & 93 & TTTTATTACT \\
\hline 19 & CTTCTGACTC & 44 & GAAGCAGGAG & 69 & CCTTTGTAAT & 94 & AGGTATCACT \\
\hline 20 & TAGTAGGCAC & 45 & CTTCCTGTTC & 70 & GCCTTTGTAA & 95 & СТСААТАТСА \\
\hline 21 & ACAGCCCATG & 46 & TAATTGGAGG & 7I & TATCAAACAC & 96 & GGTATCACTA \\
\hline 22 & CAGCCCATGC & 47 & TTCCTGTTCT & 72 & ТССТАТTACT & 97 & GTAATAATTT \\
\hline 23 & AATATAAAAC & 48 & AGTAGGCACT & 73 & CATAATTGGA & 98 & GTATCACTAT \\
\hline 24 & ATATAAAACC & 49 & TCCCCGAATA & 74 & GAGCTATTAA & 99 & TAATAATTTT \\
\hline 25 & TCTAGCAGCA & 50 & TTCCCCGAAT & 75 & GGAGCTATTA & 100 & TCAATATCAA \\
\hline
\end{tabular}

Table 10: Top-100 spectrum features for Birds of North America data set

\begin{tabular}{|c|c|c|c|c|c|c|c|}
\hline rank & feature & rank & feature & rank & feature & rank & feature \\
\hline I & ATCACAATAC & 26 & АСТTCATCAC & 51 & AAACAACATA & 76 & GATTCTTTGG \\
\hline 2 & ATAATCGGAG & 27 & AACCTAGCCC & 52 & ACAACATAAG & 77 & TATACCAACA \\
\hline 3 & ACCAACACCT & 28 & ААСТTСАTCA & 53 & ATTCTTCGAC & 78 & TAGCATTCCC \\
\hline 4 & TACCAACACC & 29 & ACCTAGCCCA & 54 & AACTGACTAG & 79 & ATAGCATTCC \\
\hline 5 & AAGCTTCTGA & 30 & ATCAACATAA & 55 & ACTGACTAGT & 80 & CGGAGCCTCA \\
\hline 6 & AACATAAGCT & 31 & ATCAACTTCA & 56 & AACAACATAA & 81 & AGACGACCAA \\
\hline 7 & ACATAAGCTT & 32 & TCAACTTCAT & 57 & AGCAATCAAC & 82 & CATGCCTTCG \\
\hline 8 & CATAAGCTTC & 33 & ATACCAAACC & 58 & СААСТTСАТС & 83 & GTAGACCTAG \\
\hline 9 & ТССТАСТССТ & 34 & СТАATCACTG & 59 & GGAGGAGACC & 84 & TAGACCTAGC \\
\hline 10 & CCССТАTTCG & 35 & СТСАСАAТАС & 60 & СТСТСАСААТ & 85 & АССССССТАТ \\
\hline II & TTCTTCGACC & 36 & ATAAGCTTCT & 61 & ACAATACCAA & 86 & ССССССТАТТ \\
\hline 12 & СССТATTCGT & 37 & TAAGCTTCTG & 62 & ACGCCGGAGC & 87 & AACCССССТA \\
\hline 13 & TCTTCGACCC & 38 & TCGTAATAAT & 63 & CACGCCGGAG & 88 & ATGCCTTCGT \\
\hline 14 & GCCTTCGTAA & 39 & CAACATAAGC & 64 & GTCCTAATCA & 89 & TCATCACAAC \\
\hline 15 & CCTTCGTAAT & 40 & GCAACCTAGC & 65 & TCСТАATCAC & 90 & TTCATCACAA \\
\hline 16 & CTTCGTAATA & 41 & GGCAACCTAG & 66 & TGATTCTTTG & 91 & AAACTGACTA \\
\hline 17 & AGCTTCTGAC & 42 & TAATCACTGC & 67 & ТССТССТССТ & 92 & АТСТTСТССС \\
\hline 18 & GCTTCTGACT & 43 & AATACCAAAC & 68 & GAGGAGACCC & 93 & ТСТТСТСССТ \\
\hline 19 & GAGCCTCAGT & 44 & САAТАCCAAA & 69 & ACATAGCATT & 94 & AAACСССССТ \\
\hline 20 & GGAGCCTCAG & 45 & ATAATTGGAG & 70 & GACATAGCAT & 95 & СAAACССССС \\
\hline 21 & CAACATAAAA & 46 & TTCGTAATAA & 71 & CAGTAGACCT & 96 & AACCTAAACA \\
\hline 22 & TCACAATACC & 47 & ACCAAACCCC & 72 & TCAGTAGACC & 97 & ACCTAAACAC \\
\hline 23 & ТССТССТАСТ & 48 & TACCAAACCC & 73 & TTCTGATTCT & 98 & CGTAATAATC \\
\hline 24 & CACAATACCA & 49 & CATAGCATTC & 74 & TCTGATTCTT & 99 & ATCGGAGGAT \\
\hline 25 & TCAACATAAA & 50 & TCTСACAATA & 75 & CATAAAACCC & 100 & TAATCTTCTT \\
\hline
\end{tabular}

corresponding value on the horizontal axis, for each of the barcode datasets. For example, in the ACG set there are about 480 species that can be predicted with the accuracy of $90 \%$ or higher. The performance of an ideal method would result in the horizontal solid-red line. In all cases, performance of the spectrum method relatively closely follows the ideal method. Majority of incorrect assignments occur on very few species, which is evident 
Table II: Top-100 spectrum features for Fish of Australia data set

\begin{tabular}{|c|c|c|c|c|c|c|c|}
\hline rank & feature & rank & feature & rank & feature & rank & feature \\
\hline I & AACATAAAAC & 26 & GACTTCTTCC & 51 & ACAGTCTACC & 76 & АТСТТСТССС \\
\hline 2 & ACATAAAACC & 27 & TAATAATTGG & 52 & CAGTCTACCC & 77 & ТСТТСТСССТ \\
\hline 3 & ATTATTAACA & 28 & AACATAAGCT & 53 & TAAATAATAT & 78 & ACTATTATTA \\
\hline 4 & TTATTAACAT & 29 & ACATAAGCTT & 54 & AGCTTCTGAC & 79 & TATTATTAAC \\
\hline 5 & TAACATAAAA & 30 & AATATCAAAC & 55 & CATAAAACCC & 80 & ATAGTAATAC \\
\hline 6 & ATTAACATAA & 31 & CAATATCAAA & 56 & CCCCGAATAA & 81 & AGGAGACCCA \\
\hline 7 & TTAACATAAA & 32 & TTATGATTGG & 57 & CCCGAATAAA & 82 & CTATTATTAA \\
\hline 8 & ТССТТСТССТ & 33 & ACCAACACCT & 58 & GCTTCTGACT & 83 & TAATATAAAA \\
\hline 9 & ATTATTAATA & 34 & TACCAACACC & 59 & TAGTAATACC & 84 & TTAATATAAA \\
\hline 10 & TTATTAATAT & 35 & TTATTACAAC & 60 & TCATGATTGG & 85 & TTGACCCTGC \\
\hline II & TATTAACATA & 36 & GAGACCCAAT & 61 & CCTCGAATAA & 86 & AATAAACAAC \\
\hline 12 & GAACAGTTTA & 37 & GGAGACCCAA & 62 & CTCGAATAAA & 87 & AATTTTATTA \\
\hline 13 & TGAACAGTTT & 38 & TTTATTACAA & 63 & СТТСТТСТСС & 88 & ATTACAATGC \\
\hline 14 & GAGGAGACCC & 39 & ATACCAATTA & 64 & AATACCAAAC & 89 & ATTTTATTAC \\
\hline 15 & GGAGGAGACC & 40 & СТTТАССАAC & 65 & САAТАCCAAA & 90 & TTACAATGCT \\
\hline 16 & TGACTTCTTC & 41 & GGAGGAGGAG & 66 & GAGGAGGAGA & 91 & TTGGAAACTG \\
\hline 17 & ATCAAACACC & 42 & TACCAATTAT & 67 & ATGAGCTTCT & 92 & TTTGACCCTG \\
\hline 18 & TATCAAACAC & 43 & TTTACCAACA & 68 & ATGATTGGAG & 93 & TTTGGAAACT \\
\hline 19 & ТТСТТСТССТ & 44 & AACAGTCTAC & 69 & TGAGCTTCTG & 94 & AATAAATAAT \\
\hline 20 & AATATAAAAC & 45 & ACAGACCGAA & 70 & TTATGATCGG & 95 & ATTAATATAA \\
\hline 21 & ATATAAAACC & 46 & CAGACCGAAA & 71 & ATAAATAATA & 96 & GAGGGGACCC \\
\hline 22 & CGAATAAATA & 47 & GAACAGTCTA & 72 & TTACCAACAC & 97 & GGAGGGGACC \\
\hline 23 & GAATAAATAA & 48 & TGAACAGTCT & 73 & ТTTССТСАAТ & 98 & AATATGAGCT \\
\hline 24 & TCTTTGACCC & 49 & ATAATTGGTG & 74 & ATATCAAACA & 99 & ACCCTGCAGG \\
\hline 25 & TTCTTTGACC & 50 & ТССТТСТТСТ & 75 & ATAATTGGAG & 100 & AGACCGAAAC \\
\hline
\end{tabular}

Table 12: Top- 100 spectrum features for Fish larvae data set

\begin{tabular}{|c|c|c|c|c|c|c|c|}
\hline rank & feature & rank & feature & rank & feature & rank & feature \\
\hline I & CGCAATCCTC & 26 & CCAGTCAATG & 51 & AATAAAGGAT & 76 & AGTGGATCAT \\
\hline 2 & GCAATCCTCT & 27 & CCCATGTGGA & 52 & AATAAATAAC & 77 & AGTTACAACT \\
\hline 3 & GCGCAATCCT & 28 & CCCCATGTGG & 53 & AATAACCCCC & 78 & ATAAACAGAA \\
\hline 4 & ATCAACGAAC & 29 & CCCCCATGTG & 54 & AATGACCCTA & 79 & ATAAAGGATT \\
\hline 5 & CGCAATCCCC & 30 & CCCCGTGCAG & 55 & AATTGATCTC & 80 & ATAAATAACC \\
\hline 6 & GATCAACGAA & 31 & CTCCCCGTGC & 56 & ACAACTCTAA & 81 & ATAACCCCCA \\
\hline 7 & GCAATCCССТ & 32 & TCCAGTCAAT & 57 & ACAAGATGGA & 82 & ATCAACGGAC \\
\hline 8 & GCGCAATCCC & 33 & TCCCCGTGCA & 58 & ACACTAAAGT & 83 & ATCATGTCAA \\
\hline 9 & TCAACGAACC & 34 & TGACCAAAAA & 59 & ACAGCTGAGA & 84 & АТССТСТTTТ \\
\hline 10 & ACCCTAGGGA & 35 & AAAAGATCCG & 60 & ACCCACCCTG & 85 & ATCGACGAGG \\
\hline II & AGTTACCCTA & 36 & AAACAGAATT & 61 & АССССТССТА & 86 & ATCTCCCCGT \\
\hline 12 & CCCTAGGGAT & 37 & AAAGATCCGG & 62 & ACCCTGATGT & 87 & ATGACCCTAA \\
\hline 13 & CCTAGGGATA & 38 & AAAGGATTGA & 63 & ACCTAGTTAC & 88 & ATGGAACCCA \\
\hline 14 & CGATCAACGA & 39 & AAAGTGGATC & 64 & ACGGACCTAG & 89 & ATGTCAATGA \\
\hline 15 & GTTACCCTAG & 40 & AAATAAAGGA & 65 & ACTAAAGTGG & 90 & ATGTGGAATG \\
\hline 16 & TACCCTAGGG & 41 & AAATAACCCC & 66 & АСТСТААТАA & 91 & ATTGAACAAG \\
\hline 17 & TTACCCTAGG & 42 & AACAAGATGG & 67 & AGAAGCGGGG & 92 & ATTGATCTCC \\
\hline 18 & TCTGACCAAT & 43 & AACCCACCСТ & 68 & AGACACTAAA & 93 & CAACGGACCT \\
\hline 19 & TTTCAAGTCA & 44 & AACGGACCTA & 69 & AGAGTCCATA & 94 & СААСТСТААТ \\
\hline 20 & CTGACCAAAA & 45 & AACTCTAATA & 70 & AGAGTTACAA & 95 & CAAGATGGAA \\
\hline 21 & TCTGACCAAA & 46 & AAGATGGAAC & 71 & AGATGGAACC & 96 & СААТССССТС \\
\hline 22 & AAACTAAGAG & 47 & AAGCGGGGAT & 72 & AGCGGGGATT & 97 & СААТССТСТТ \\
\hline 23 & AACCCCCATG & 48 & AAGGATTGAA & 73 & AGCTGAGAGT & 98 & CAATGACCCT \\
\hline 24 & ACCCCCATGT & 49 & AAGTGGATCA & 74 & AGGATTGAAC & 99 & САССССТССТ \\
\hline 25 & CAGTCAATGA & 50 & AATAAACAGA & 75 & AGTCCATATC & 100 & CACCCTGATG \\
\hline
\end{tabular}

from the sudden initial drops in the curves. We observe that incorrect assignments of the samples from new species to the existing classes often resulted from the presence of the (nearly) duplicate sequences in the training set. For instance, in the Astraptes dataset, query sequences from SENNOV were closest to an identical sequence which is also present in the YESENN species. This points to the need for alternative sources of 
Table 13: New species detection (average per-class error, \%)

\begin{tabular}{lcc}
\hline Dataset & error & \# classes with 0\% error \\
\hline ACG & 14.12 & $474 / 573$ \\
Hesperiidae & 17.53 & $288 / 364$ \\
Astraptes & 7.81 & $10 / 12$ \\
Bats of Guyana & 5.80 & $90 / 96$ \\
Birds of North America & 16.36 & $524 / 656$ \\
Fish of Australia & 16.21 & $174 / 211$ \\
\hline
\end{tabular}

Table 14: New species detection (average error rate, \%)

\begin{tabular}{lc}
\hline Dataset & Error \\
\hline ACG & 10.29 \\
Hesperiidae & 10.88 \\
Astraptes & 8.47 \\
Bats of Guyana & 9.95 \\
Birds of North America & 15.54 \\
Fish of Australia & 14.92 \\
Fish larvae & 15.77 \\
\hline
\end{tabular}

information in cases where the barcodes may not be sufficient to discriminate among species but also to the need for additional curation in some of the datasets.

\section{Barcode clustering}

In this section, we consider the problem of arranging barcode sequences into groups (clusters) to establish relationships between sequences and automatically discover class structure. Clustering can be useful for putative samples with no prior evidence of species assignments. Clustering-based evaluation also allows one to eliminate the influence of the classification training (weight estimation) process, which is an additional factor that affects the classification performance. As a consequence, clustering may provide a more direct insight into the quality of the similarity measure alone.

To evaluate the results of clustering we compare the partitioning obtained by the clustering model with the partitioning given by the known species membership. We perform clustering experiments using the recently proposed affinity propagation algorithm [39] which makes use of the computed similarity scores. We report clustering results using the alignment-free methods only in Table 15, in the light of the classification results which we presented earlier across different similarity metrics. As we can see from the table, clustering of barcode datasets results in accurate partitioning of the barcodes into groups that are similar to

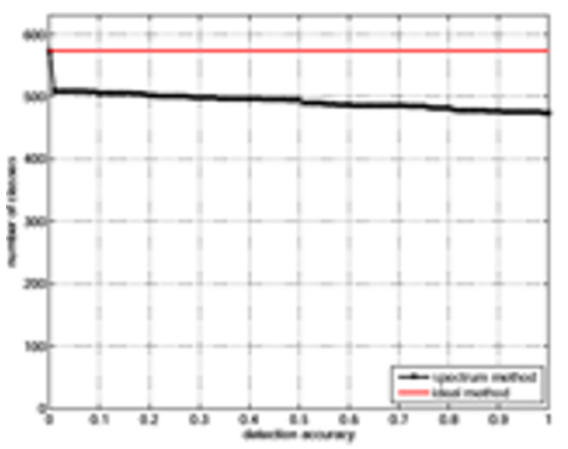

(a) ACG

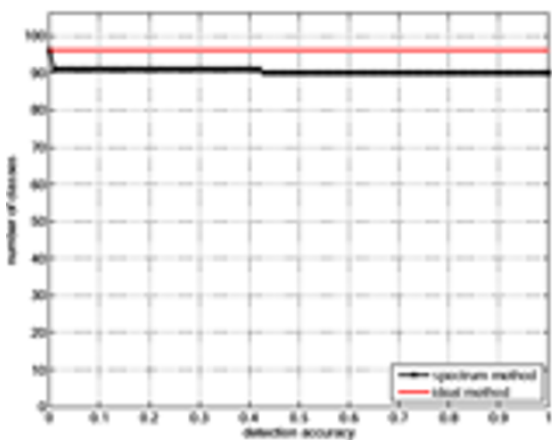

(d) Bats of Guyana

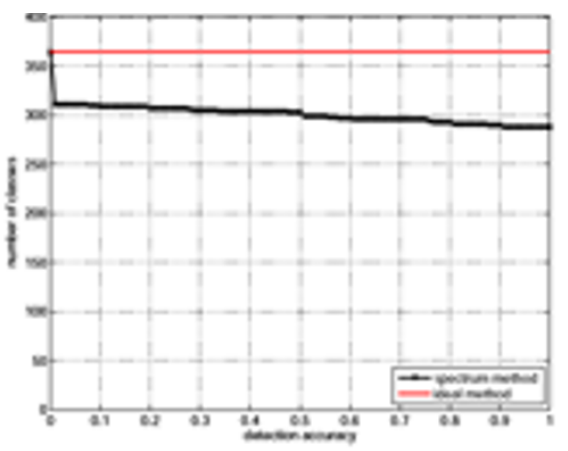

(b) Hesperiidae

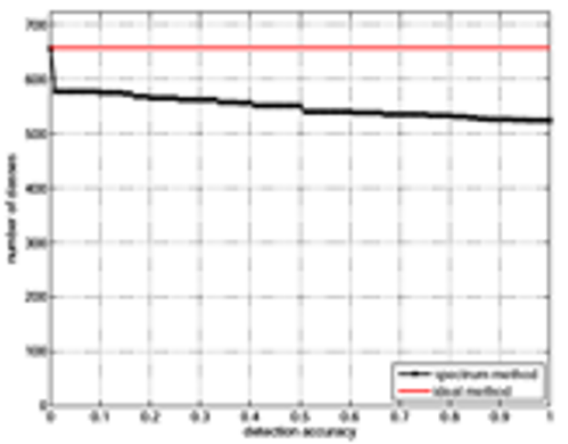

(e) Birds of North America

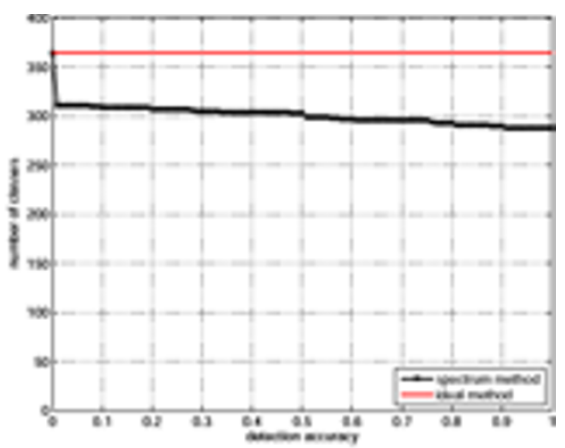

(c) Astraptes

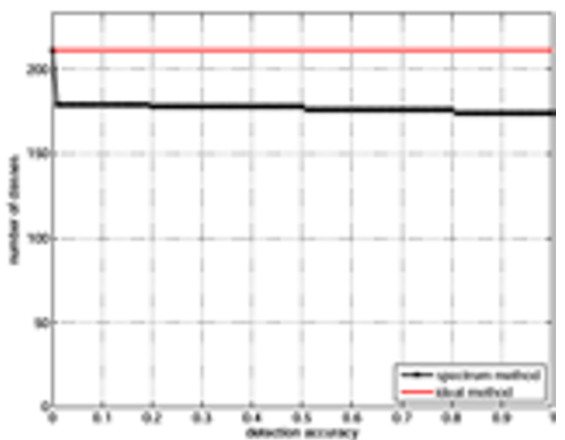

(f) Fish of Australia

Figure I I

New species detection performance. 
Table I5: Clustering results (using spectrum similarity measure)

\begin{tabular}{lcccc}
\hline Dataset & \#clusters & error, $\%$ & Rand index & Jaccard index \\
\hline ACG & 644 & 2.84 & 99.85 & 83.96 \\
Hesperiidae & 382 & 4.44 & 99.79 & 86.42 \\
Astraptes & 17 & 1.51 & 95.59 & 81.59 \\
Bats & 98 & 0.95 & 99.21 & 86.58 \\
Birds & 650 & 5.25 & 99.90 & 86.59 \\
Fish Australia & 235 & 2.52 & 99.94 & 93.07 \\
Fish larvae & 7 & 2.86 & 98.66 & 95.51 \\
\hline
\end{tabular}

the true species-induced classes. We use Rand [40] and Jaccard indices to evaluate the clustering quality. Rand $=(a+$ d) $/ T$, Jaccard $=a /(a+b+c)$, where $a$ is the number pairs with the same class label assigned to the same cluster, $b$ is the number of points with the same class label assigned to different clusters, $c$ is the number of pairs with different class labels placed into the same cluster, $d$ is the number of pairs with different labels placed into different clusters, and $T$ is the total number of pairs.

To further illustrate the clustering results, we show in Figure 12 projections of the barcode data onto a $2 \mathrm{D}$ plane. We note the agreement of species labels and proximities in the embedded space. This suggests possibility of using clustering with alignment-free methods as an efficient and accurate tool for exploratory analysis of newly obtained barcodes.

\section{Experimental running time analysis}

Computational complexity of evaluating similarity on large sets of barcodes may be a prominent factor in

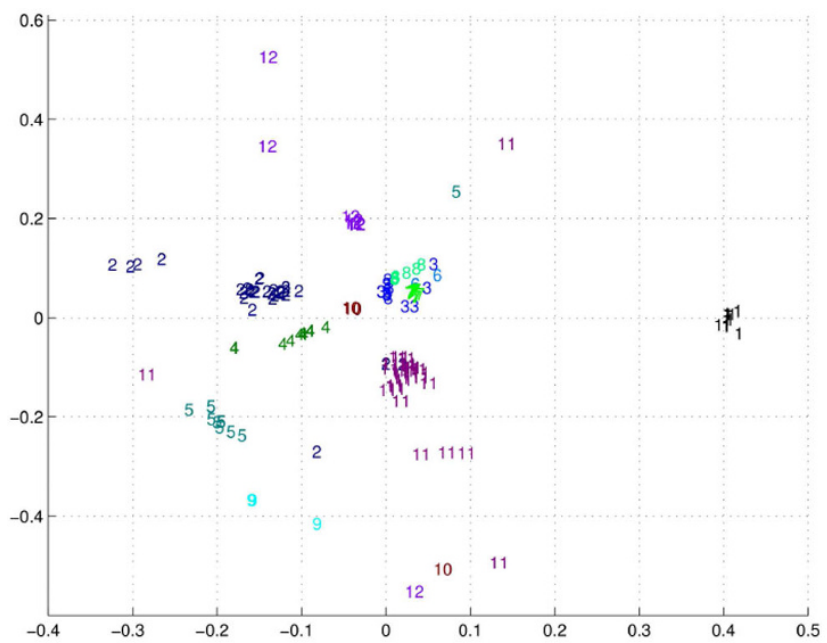

(a) Clustering (projection in 2D) on Astraptes data set practical barcoding applications. We evaluate the computational speed of different methods by measuring running time for computing similarity scores among all pairs of sequences in a barcode dataset. The running times are obtained by running the optimized versions of all targeted methods on a single $3.0 \mathrm{GHz}$ quad core CPU using 2 GB RAM (Dell PowerEdge 2950). Table 16 compares running times for the alignment-free kernel methods as well as PSI-BLAST and alignment-based (Smith-Waterman) methods. As we can see from the table, alignment-free kernel methods are significantly faster compared to computationally demanding alignment-based (Smith-Waterman) methods. For example, it takes about 60 seconds to evaluate 4267-by-4267 similarity matrix using the spectrum method compared to 64800 seconds $(18 \mathrm{~h})$ for computing the SmithWaterman scoring matrix. These results are not surprising given the complexity analysis mentioned in Section 'Methods' and further studied in [31]. The low computational complexity of the alignment-free scores, besides its appeal for analytics on large datasets, also opens the

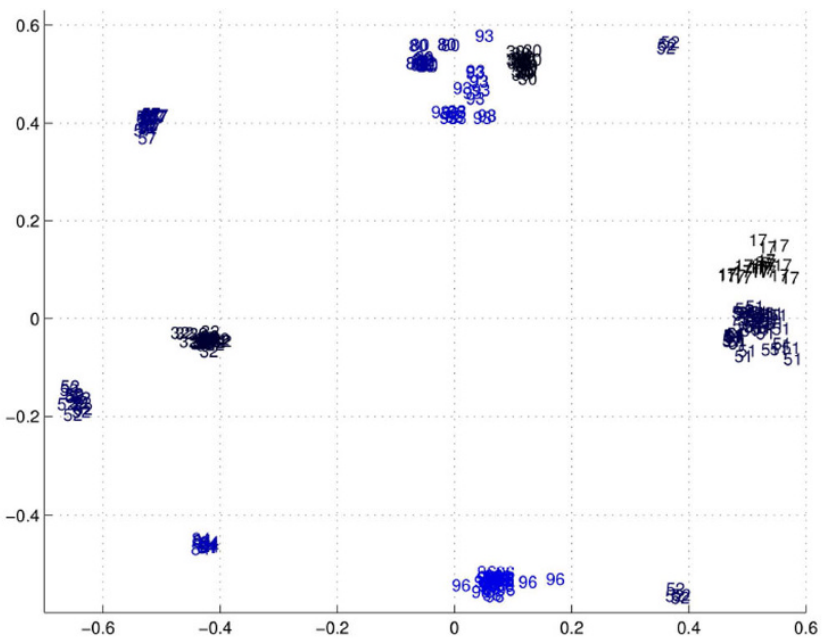

(b) Clustering (projection in 2D) on Bats of Guyana data set (for clarity, only 10 largest classes are shown)

Figure 12

Clustering results for barcode sequences using alignment-free spectrum method (data is projected onto 2D plane). 
Table 16: Running time (kernel computation), s

\begin{tabular}{lllll}
\hline Dataset & PSI-BLAST & Smith-Waterman & spectrum (k= 10) & mismatch \\
\hline ACG & 2557 & 64818 & 60.328 & 366.39 \\
Hesperiidae & 891 & 19378 & 16.16 & 90.26 \\
Astraptes & 99 & 854 & 0.89 & 2.15 \\
Bats & 239 & 2818 & 1.91 & 12.12 \\
Birds & 1147 & 27311 & 18.75 & 149.67 \\
Fish Australia & 203 & 3424 & 1.57 & 9.95 \\
Fish larvae & 1.9 & 39.58 & 0.23 & 0.16 \\
\hline
\end{tabular}

possibility for using these metrics on conceptualized handheld barcode scanners that may be designed in the future.

\section{Conclusion}

In this work we demonstrate that newly developed alignment-free methods can serve as efficient and accurate analytical tools for DNA barcoding problems. The new alignment-free methods provide highly accurate and computationally efficient identification and classification of barcode sequences as we show on a set of various barcode collections. Using new alignment-free scoring approaches demonstrates excellent performance in comparison with more computationally demanding, traditional alignment-based methods. The use of alignment-free scoring methods allows discovery of natural groups (clusters) in barcode collections that accurately reflect the species-based groupings. This reflects potentially high agreement between the proposed fragmentinduced sequence similarity measures and the within and across species barcode diversity. Finally, we show that the spectral methods also foster discovery of withinbarcode markers that point to critical differences among barcodes of different sample groups. These markers can serve both as the sparse and robust barcode codes and as possible pointers to within barcode loci that deserve further investigation. Our experiments finally suggest that it may be possible to further improve the performance of our spectral scores by merging them with the position-based metrics, such as the Kimura distance. Currently, the spectral kernels rely on the $0 / 1$ scoring within each fragment. While implementing Kimura scoring within $k$-mers is possible, a direct implementation adversely affects the spectral algorithm's efficiency, with the complexity becoming quadratic in the sequence length. This leaves open an avenue for future research into efficient spectral barcoding algorithms for arbitrary fragment scores.

\section{Competing interests}

The authors declare that they have no competing interests.

\section{Authors' contributions}

All authors contributed equally to this work.

\section{Acknowledgements}

This article has been published as part of BMC Bioinformatics Volume 10 Supplement 14, 2009: Biodiversity Informatics. The full contents of the supplement are available online at http://www.biomedcentral.com/I47I$2105 / 10$ ? issue $=\mathrm{S} \mid 4$.

\section{References}

I. Hebert PDN, Cywinska A, Ball S and deWaard J: Biological identifications through DNA barcodes. Proceedings of the Royal Society of London 2003, 3/3-322.

2. Stoeckle $M$ and Herbert PDN: Barcode of Life. Scientific American 2008, 82-88.

3. Linares $M$, Soto-Calderón I, Lees $D$ and Anthony $N$ : High mitochondrial diversity in geographically widespread butterflies of Madagascar: A test of the DNA barcoding approach. Mol Phylogenet Evol 2008.

4. Armstrong KF and Ball SL: DNA barcodes for biosecurity: invasive species identification. Philos Trans $R$ Soc Lond, B, Biol Sci 2005, 360(1462): 1813-23.

5. Smith P, McVeagh S, Steinke D and Meyer A: DNA barcoding for the identification of smoked fish products. Journal of Fish Biology 2008.

6. Wong $E$ and Hanner R: DNA barcoding detects market substitution in North American seafood. Food Research International 2008.

7. Holmes B, Steinke D and Ward R: Identification of shark and ray fins using DNA barcoding. Fisheries Research 2008.

8. Saunders G: Applying DNA barcoding to red macroalgae: a preliminary appraisal holds promise for future .... Philosophical Transactions of the Royal Society B: ... 2005.

9. Sogin ML, Morrison HG, Huber JA, Welch DM, Huse SM, Neal PR, Arrieta JM and Herndl GJ: Microbial diversity in the deep sea and the underexplored "rare biosphere". Proceedings of the National Academy of Sciences 2006, 103(32):121/5-12120 http:// www.pnas.org/content/103/32/12115.abstract.

10. Kress W, Wurdack K, Zimmer E and Weigt L: Use of DNA barcodes to identify flowering plants. Proceedings of the National Academy of Sciences 2005 http://www.pnas.org/cgi/content/abstract/ 102/23/8369.

I I. Chase MW, Salamin N, Wilkinson M, Dunwell JM, Kesanakurthi RP, Haidar N and Savolainen V: Land plants and DNA barcodes: short-term and long-term goals. Philos Trans $R$ Soc Lond, B, Biol Sci 2005, 360( (1462): 1889-95.

12. Kress WJ and Erickson DL: DNA Barcoding-a Windfall for Tropical Biology?. BIOTROPICA 2008.

13. Barrett $R$ and Hebert $P$ : Identifying spiders through DNA barcodes. Can J Zool 2005, 83(3):48|-49I.

14. Ward RD, Zemlak TS, Innes BH, Last PR and Hebert PD: DNA barcoding Australia's fish species. Philosophical Transactions of the Royal Society B: Biological Sciences 2005, 360( (462): | 847-1857.

15. Robins J, Hingston M, Matisoo-Smith $E$ and Ross $\mathrm{H}$ : Identifying Rattus species using mitochondrial DNA. Molecular Ecology Notes 2007 .

16. Steinke $D$, Vences M, Salzburger $W$ and Meyer A: Taxl: a software tool for DNA barcoding using distance methods. Philosophical Transactions of the Royal Society B: Biological Sciences 2005, 360 ( I 462): 1975-1980.

17. Abdo $Z$ and Golding GB: A step toward barcoding life: a modelbased, decision-theoretic method to assign genes to preexisting species groups. Systematic Biology 2007, 56:44-56.

18. Nielsen R and Matz M: Statistical Approaches for DNA Barcoding. Systematic Biology 2006, 55:162-169. 
19. Zhang A: Inferring Species Membership Using DNA Sequences with Back-Propagation Neural Networks. Systematic Biology 2008.

20. Matz MV and Nielsen R: A likelihood ratio test for species membership based on DNA sequence data. Philosophical Transactions of the Royal Society B: Biological Sciences 2005, 360 (I462): I969-1974.

2I. Meyer CP and Paulay G: DNA barcoding: error rates based on comprehensive sampling. PLoS Biol 2005, 3(I 2):

22. Leslie CS, Eskin E and Noble WS: The Spectrum Kernel: $\mathbf{A}$ String Kernel for SVM Protein Classification. Pacific Symposium on Biocomputing 2002, 566-575.

23. Leslie CS, Eskin E, Weston J and Noble WS: Mismatch String Kernels for SVM Protein Classification. NIPS MIT Press: Becker S, Thrun S, Obermayer K 2002, |4|7-|424.

24. Kuang R, le E, Wang K, Wang K, Siddiqi M, Freund $Y$ and Leslie C: Profile-Based String Kernels for Remote Homology Detection and Motif Extraction. CSB '04: Proceedings of the 2004 IEEE Computational Systems Bioinformatics Conference (CSB'04) Washington, DC, USA: IEEE Computer Society; 2004, I 52-160.

25. Jaakkola T, Diekhans $M$ and Haussler D: A Discriminative Framework for Detecting Remote Protein Homologies. Journal of Computational Biology 2000, 7(I-2):95-I| 4.

26. Menchetti S, Costa F and Frasconi P: Weighted decomposition kernels. ICML '05: Proceedings of the 22nd international conference on Machine learning New York, NY, USA: ACM Press; 2005, 585-592.

27. Schölkopf B and Smola AJ: Learning with kernels MIT Press; 2002.

28. Vapnik V: Statistical learning theory Wiley; 1998.

29. Leslie CS, Eskin E, Weston J and Noble WS: Mismatch String Kernels for SVM Protein Classification. NIPS 2002, |4I7-|424.

30. Leslie $\mathrm{C}$ and Kuang R: Fast String Kernels using Inexact Matching for Protein Sequences. J Mach Learn Res 2004, 5:1435-1455.

31. Kuksa P, Huang PH and Pavlovic V: Scalable Algorithms for String Kernels with Inexact Matching. NIPS 2008.

32. Allwein EL, Schapire RE and Singer Y: Reducing multiclass to binary: a unifying approach for margin classifiers. J Mach Learn Res 2001, I:I13-141.

33. http://www.boldsystems.org.

34. http://dimacs.rutgers.edu/Workshops/BarcodeResearchChallenges2007/.

35. http://www.kyb.tuebingen.mpg.de/bs/people/spider.

36. http://seqam.rutgers.edu/projects/bioinfo/barcoding/barcoding.html.

37. Hebert PDN, Penton EH, Burns JM, Janzen DH and Hallwachs W: Ten species in one: DNA barcoding reveals cryptic species in the neotropical skipper butterfly Astraptes fulgerator. PNAS 2004, I01:|4812-14817.

38. Kononenko I: Estimating attributes: analysis and extensions of RELIEF. ECML-94: Proceedings of the European conference on machine learning on Machine Learning Secaucus, NJ, USA: Springer-Verlag New York, Inc; 1994, I7I-182.

39. Frey BJ and Dueck D: Clustering by Passing Messages Between Data Points. Science 2007, 3 I5:972-976 http://www.psi.toronto. edu/affinitypropagation.

40. Rand WM: Objective Criteria for the Evaluation of Clustering Methods. Journal of the American Statistical Association 1971, 66 (336):846-850 http://www.jstor.org/stable/2284239.
Publish with BioMed Central and every scientist can read your work free of charge

"BioMed Central will be the most significant development for disseminating the results of biomedical research in our lifetime. "

Sir Paul Nurse, Cancer Research UK

Your research papers will be:

- available free of charge to the entire biomedical community

- peer reviewed and published immediately upon acceptance

- cited in PubMed and archived on PubMed Central

- yours - you keep the copyright
BioMedcentral 